Article

\title{
The Logic of Pseudo-Uninorms and Their Residua
}

\section{SanMin Wang}

Faculty of Science, Zhejiang Sci-Tech University, Hangzhou 310018, China; wangsanmin@hotmail.com; Tel.: +86-136-5581-2543

Received: 15 February 2019; Accepted: 8 March 2019; Published: 12 March 2019

Abstract: Our method for density elimination is generalized to the non-commutative substructural logic $\mathrm{GpsUL}^{*}$. Then, the standard completeness of $\mathbf{H p s U L}^{*}$ follows as a lemma by virtue of previous work by Metcalfe and Montagna. This result shows that HpsUL ${ }^{*}$ is the logic of pseudo-uninorms and their residua and answered the question posed by Prof. Metcalfe, Olivetti, Gabbay and Tsinakis.

Keywords: density elimination; pseudo-uninorm logic; standard completeness of $\mathbf{H p s U L}^{*}$; substructural logics; fuzzy logic

MSC: 03B50; 03F05; 03B52; 03B47

\section{Introduction}

Prof. Metcalfe, Olivetti and Gabbay conjectured that the Hilbert system HpsUL is the logic of pseudo-uninorms and their residua in 2009 in [1]. It is not the case, as shown by Prof. Wang and Zhao in [2], although HpsUL is the logic of bounded representable residuated lattices. We constructed the system $\mathrm{HpsUL}^{*}$ by adding the weakly commutativity rule

$$
(W C M) \vdash(A \leadsto t) \rightarrow(A \rightarrow t)
$$

to HpsUL and conjectured that it is the logic of residuated pseudo-uninorms and their residua in 2013 in [3].

In this paper, we prove the conjecture by showing that the density elimination holds for the hypersequent system $\mathrm{GpsUL}^{*}$ corresponding to $\mathrm{HpsUL}^{*}$. Then, the standard completeness of HpsUL $^{*}$ follows as a lemma by virtue of previous work by Metcalfe and Montagna [4]. That is, $\mathrm{HpsUL}^{*}$ is complete with respect to algebras whose lattice reduct is the real unit interval $[0,1]$. Thus, $\mathbf{H p s U L}^{*}$ is a kind of substructural fuzzy logic [4], and potentially has certain applications to fuzzy inferences and expert Systems [5-8]. Our result also shows that that $\mathbf{H p s U L}^{*}$ is an axiomatization for the variety of residuated lattices generated by all dense residuated chains. Thus, we have also answered the question posed by Prof. Metcalfe and Tsinakis in [9] in 2017.

In proving the density elimination for $\mathrm{GpsUL}^{*}$, we have to overcome several difficulties as follows. Firstly, cut-elimination doesn't holds for $\mathbf{G p s U L}^{*}$. Note that (WCM) and the density rule $(D)$ are formulated as

$$
\frac{G \mid \Gamma, \Delta \Rightarrow t}{G \mid \Delta, \Gamma \Rightarrow t}, \quad \frac{G|\Pi \Rightarrow p| \Gamma, p, \Delta \Rightarrow B}{G \mid \Gamma, \Pi, \Delta \Rightarrow B}
$$

in $\mathrm{GpsUL}^{*}$, respectively. Consider the following derivation fragment.

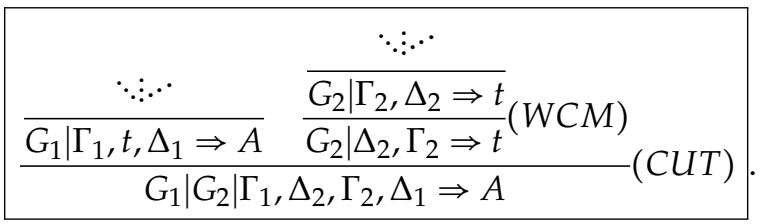


By the induction hypothesis of the proof of cut-elimination, we get that $G_{1}\left|G_{2}\right| \Gamma_{1}, \Gamma_{2}, \Delta_{2}, \Delta_{1} \Rightarrow A$ from $G_{2} \mid \Gamma_{2}, \Delta_{2} \Rightarrow t$ and $G_{1} \mid \Gamma_{1}, t, \Delta_{1} \Rightarrow A$ by $(C U T)$. However, we can't deduce $G_{1}\left|G_{2}\right| \Gamma_{1}, \Delta_{2}, \Gamma_{2}, \Delta_{1} \Rightarrow A$ from $G_{1}\left|G_{2}\right| \Gamma_{1}, \Gamma_{2}, \Delta_{2}, \Delta_{1} \Rightarrow A$ by $(W C M)$. We overcome this difficulty by introducing the following weakly cut rule into $\mathrm{GpsUL}^{*}$

$$
\frac{G_{1}\left|\Gamma, t, \Delta \Rightarrow A \quad G_{2}\right| \Pi \Rightarrow t}{G_{1}\left|G_{2}\right| \Gamma, \Pi, \Delta \Rightarrow A}(W C T) .
$$

Secondly, the proof of the density elimination for $\mathrm{GpsUL}^{*}$ becomes troublesome even for some simple cases in GUL [4]. Consider the following derivation fragment

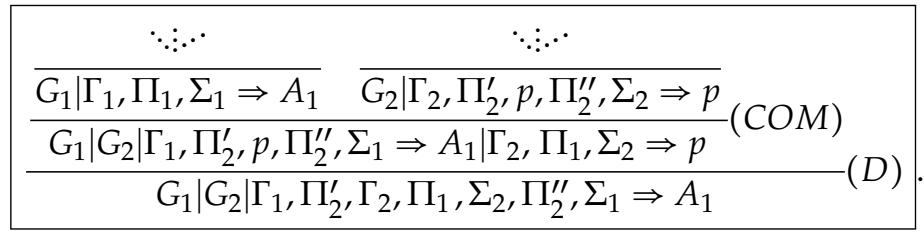

Here, the major problem is how to extend $(D)$ such that it is applicable to $G_{2} \mid \Gamma_{2}, \Pi_{2}^{\prime}, p, \Pi_{2}^{\prime \prime}, \Sigma_{2} \Rightarrow p$. By replacing $p$ with $t$, we get $G_{2} \mid \Gamma_{2}, \Pi_{2}^{\prime}, t, \Pi_{2}^{\prime \prime}, \Sigma_{2} \Rightarrow t$. However, there exists no derivation of $G_{1}\left|G_{2}\right| \Gamma_{1}, \Pi_{2}^{\prime}, \Gamma_{2}, \Pi_{1}, \Sigma_{2}, \Pi_{2}^{\prime \prime}, \Sigma_{1} \Rightarrow A_{1}$ from $G_{2} \mid \Gamma_{2}, \Pi_{2}^{\prime}, \Pi_{2}^{\prime \prime}, \Sigma_{2} \Rightarrow t$ and $G_{1} \mid \Gamma_{1}, \Pi_{1}, \Sigma_{1} \Rightarrow A_{1}$. Notice that $\Gamma_{2}, \Pi_{2}^{\prime}$ and $\Pi_{2}^{\prime \prime}, \Sigma_{2}$ in $G_{2} \mid \Gamma_{2}, \Pi_{2}^{\prime}, p, \Pi_{2}^{\prime \prime}, \Sigma_{2} \Rightarrow p$ are commutated simultaneously in $G_{1}\left|G_{2}\right| \Gamma_{1}, \Pi_{2}^{\prime}, \Gamma_{2}, \Pi_{1}, \Sigma_{2}, \Pi_{2}^{\prime \prime}, \Sigma_{1} \Rightarrow A_{1}$, which we can't obtain by (WCM). It seems that (WCM) can't be strengthened further in order to solve this difficulty. We overcome this difficulty by introducing a restricted subsystem $\mathrm{GpsUL}_{\Omega}$ of $\mathrm{GpsUL}^{*} . \mathrm{GpsUL}_{\Omega}$ is a generalization of $\mathbf{G I U L}_{\Omega}$, which we introduced in [10] in order to solve a longstanding open problem, i.e., the standard completeness of IUL. Two new manipulations, which we call the derivation-splitting operation and derivation-splicing operation, are introduced in $\mathrm{GpsUL}_{\Omega}$.

The third difficulty we encounter is that the conditions of applying the restricted external contraction rule $\left(E C_{\Omega}\right)$ become more complex in $\mathrm{GpsUL}_{\Omega}$ because new derivation-splitting operations make the conclusion of the generalized density rule to be a set of hypersequents rather than one hypersequent. We continue to apply derivation-grafting operations in the separation algorithm of the multiple branches of GIUL $_{\Omega}$ in [10], but we have to introduce a new construction method for $\mathrm{GpsUL}_{\Omega}$ by induction on the height of the complete set of maximal ( $\left.p E C\right)$-nodes rather than on the number of branches.

The structure of this paper is as follows. In Section 2, we present two hypersequent calculi GpsUL ${ }^{*}$ and $\mathrm{GpsUL}_{\Omega}$, and prove that Cut-elimination does not hold for GpsUL ${ }^{*}$. Because of the absence of the commutativity rule, we have to introduce two novel operations, i.e., the derivation-splitting operation and derivation-splicing operation, in $\mathrm{GpsUL}_{\Omega}$ in Section 3, and then we present a suitable definition of the generalized density rule $(\mathcal{D})$ for $\mathrm{GpsUL}_{\Omega}$. In Section 4 , we adapt the old main algorithm in the system GIUL $_{\Omega}$ to the new system $\mathrm{GpsUL}_{\Omega}$. In Section 5, we propose two directions for future research.

2. GpsUL, GpsUL ${ }^{*}$ and $\mathrm{GpsUL}_{\Omega}$

Definition 1. ([1]) GpsUL consists of the following initial sequents and rules: Initial sequents

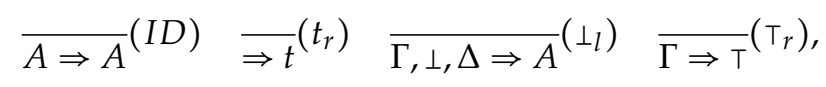

Structural Rules

$$
\frac{G|\Gamma \Rightarrow A| \Gamma \Rightarrow A}{G \mid \Gamma \Rightarrow A}(E C) \quad \frac{G}{G \mid \Gamma \Rightarrow A}(E W)
$$




$$
\frac{G_{1}\left|\Gamma_{1}, \Pi_{1}, \Delta_{1} \Rightarrow A_{1} \quad G_{2}\right| \Gamma_{2}, \Pi_{2}, \Delta_{2} \Rightarrow A_{2}}{G_{1}\left|G_{2}\right| \Gamma_{1}, \Pi_{2}, \Delta_{1} \Rightarrow A_{1} \mid \Gamma_{2}, \Pi_{1}, \Delta_{2} \Rightarrow A_{2}}(C O M)
$$

\section{Logical Rules}

$$
\begin{array}{r}
G_{1}\left|\Gamma \Rightarrow A \quad G_{2}\right| \Delta \Rightarrow B \\
G_{1}\left|G_{2}\right| \Gamma, \Delta \Rightarrow A \odot B
\end{array}\left(\odot_{r}\right)
$$

$$
\begin{gathered}
\frac{G \mid \Gamma, A, B, \Delta \Rightarrow C}{G \mid \Gamma, A \odot B, \Delta \Rightarrow C}\left(\odot_{l}\right) \\
\frac{G \mid A, \Gamma \Rightarrow B}{G \mid \Gamma \Rightarrow A \rightarrow B}\left(\rightarrow_{r}\right) \\
\frac{G \mid \Gamma, A \Rightarrow B}{G \mid \Gamma \Rightarrow A \sim B}\left(\sim_{r}\right) \\
\frac{G \mid \Gamma \Rightarrow A}{G \mid \Gamma \Rightarrow A \vee B}\left(\vee_{r r}\right) \\
\frac{G \mid \Gamma \Rightarrow B}{G \mid \Gamma \Rightarrow A \vee B}\left(\vee_{r l}\right) \\
\frac{G \mid \Gamma, B, \Delta \Rightarrow C}{G \mid \Gamma, A \wedge B, \Delta \Rightarrow C}\left(\wedge_{r l}\right) .
\end{gathered}
$$

Cut Rule

$$
\frac{G_{1}\left|\Gamma, A, \Delta \Rightarrow B \quad G_{2}\right| \Pi \Rightarrow A}{G_{1}\left|G_{2}\right| \Gamma, \Pi, \Delta \Rightarrow B}(C U T) .
$$

Definition 2. ([3]) GpsUL ${ }^{*}$ is GpsUL plus the weakly commutativity rule

$$
\frac{G \mid \Gamma, \Delta \Rightarrow t}{G \mid \Delta, \Gamma \Rightarrow t}(W C M)
$$

Definition 3. GpsUL ${ }^{* D}$ is GpsUL $^{*}$ plus the density rule $\frac{G|\Pi \Rightarrow p| \Gamma, p, \Delta \Rightarrow B}{G \mid \Gamma, \Pi, \Delta \Rightarrow B}(D)$.

Lemma 1. $G \equiv B \vee((D \rightarrow B) \odot C \odot(C \rightarrow D) \odot A \rightarrow A)$ is not a theorem in HpsUL.

Proof. Let $\mathcal{A}=(\{0,1,2,3\}, \wedge, \vee, \odot, \rightarrow, \sim, 2,0,3)$ be an algebra, where $x \wedge y=\min (x, y), x \vee y=\max (x, y)$ for all $x, y \in\{0,1,2,3\}$, and the binary operations $\odot, \rightarrow$ and $\sim$ are defined by the following tables (see [2]).

\begin{tabular}{|l|l|l|l|l|}
\hline$\odot$ & 0 & 1 & 2 & 3 \\
\hline 0 & 0 & 0 & 0 & 0 \\
\hline 1 & 0 & 1 & 1 & 1 \\
\hline 2 & 0 & 1 & 2 & 3 \\
\hline 3 & 0 & 3 & 3 & 3 \\
\hline
\end{tabular}

\begin{tabular}{|r|l|l|l|l|}
\hline$\rightarrow$ & 0 & 1 & 2 & 3 \\
\hline 0 & 3 & 3 & 3 & 3 \\
\hline 1 & 0 & 3 & 3 & 3 \\
\hline 2 & 0 & 1 & 2 & 3 \\
\hline 3 & 0 & 0 & 0 & 3 \\
\hline
\end{tabular}

\begin{tabular}{|c|c|c|c|c|}
\hline$\sim$ & 0 & 1 & 2 & 3 \\
\hline 0 & 3 & 3 & 3 & 3 \\
\hline 1 & 0 & 2 & 2 & 3 \\
\hline 2 & 0 & 1 & 2 & 3 \\
\hline 3 & 0 & 1 & 1 & 3 \\
\hline
\end{tabular}

By easy calculation, we get that $\mathcal{A}$ is a linearly ordered HpsUL-algebra, where 0 and 3 are the least and the greatest element of $\mathcal{A}$, respectively, and 2 is its unit. Let $v(A)=v(B)=v(C)=v(D)=1$. Then, $v(G)=1 \vee(3 \odot 1 \odot 3 \odot 1 \rightarrow 1)=1<2$. Hence, $G$ is not a tautology in HpsUL. Therefore, it is not a theorem in HpsUL by Theorem 9.27 in [1].

Theorem 1. Cut-elimination doesn't hold for GpsUL ${ }^{*}$.

Proof. $G \equiv \Rightarrow B \vee((D \rightarrow B) \odot C \odot(C \rightarrow D) \odot A \rightarrow A)$ is provable in $\mathbf{G p s U L}^{*}$, as shown in Figure 1. 


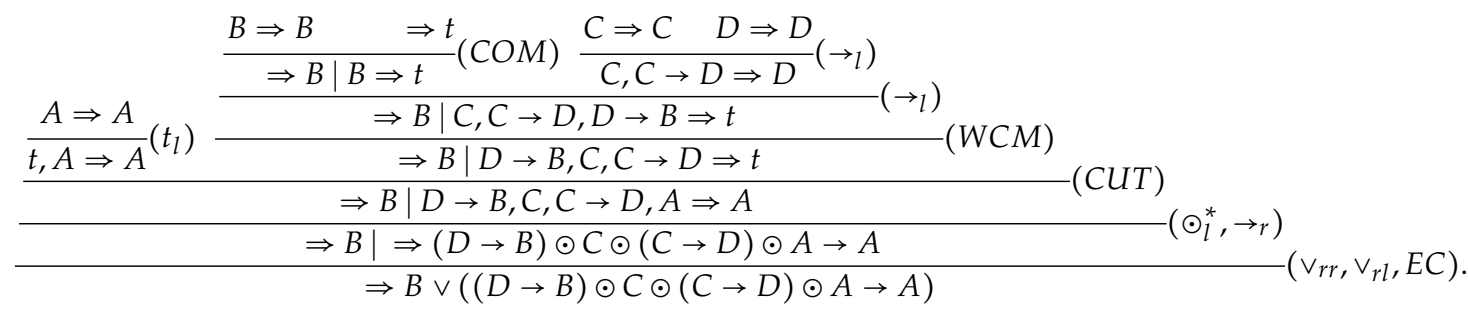

Figure 1. A proof $\tau$ of $G$.

Suppose that $G$ has a cut-free proof $\rho$. Then, there exists no occurrence of $t$ in $\rho$ by its subformula property. Thus, there exists no application of (WCM) in $\rho$. Hence, G is a theorem of GpsUL, which contradicts Lemma 1.

Remark 1. Following the construction given in the proof of Theorem 53 in [4], (CUT) in Figure 1 is eliminated by the following derivation, as shown in Figure 2. However, the application of (WCM) in $\rho$ is invalid, which illustrates the reason why the cut-elimination theorem doesn't hold in GpsUL*.

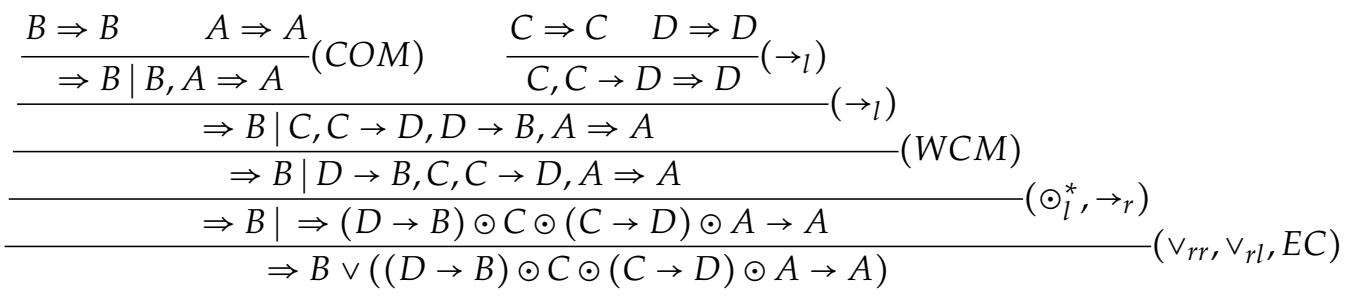

Figure 2. A possible cut-free proof $\rho$ of $G$.

Definition 4. GpsUL ${ }^{*}$ is constructed by replacing (CUT) in $\mathrm{GpsUL}^{*}$ with

$$
\frac{G_{1}\left|\Gamma, t, \Delta \Rightarrow A \quad G_{2}\right| \Pi \Rightarrow t}{G_{1}\left|G_{2}\right| \Gamma, \Pi, \Delta \Rightarrow A}(W C T) .
$$

We call it the weakly cut rule and denote it by (WCT).

Theorem 2. If $\vdash_{\mathrm{GpsUL}^{*}} \mathrm{G}$, then $\vdash_{\mathrm{GpsUL}}{ }^{*} \mathrm{G}$.

Proof. It is proved by a procedure similar to that of Theorem 53 in [4] and omitted.

Definition 5. ( [10]) $\mathrm{GpsUL}_{\Omega}$ is a restricted subsystem of $\mathbf{G p s U L}^{*}$ such that

(i) $p$ is designated as the unique eigenvariable by which we mean that it is not used to build up any formula containing logical connectives and is only used as a sequent-formula.

(ii) Each occurrence of $p$ in a hypersequent is assigned one unique identification number $i$ in $\mathbf{G}_{\mathbf{p s}} \mathrm{UL}_{\Omega}$ and written as $p_{i}$. Initial sequent $p \Rightarrow p$ of $\mathbf{G p s U L}{ }^{*}$ has the form $p_{i} \Rightarrow p_{i}$ in $\mathbf{G} \mathbf{p s} \mathbf{U L}_{\Omega}$. $p$ doesn't occur in $A, \Gamma$ or $\Delta$ for each initial sequent $\Gamma, \perp, \Delta \Rightarrow A$ or $\Gamma \Rightarrow T$ in $\mathbf{G p s}_{\Omega} \mathbf{U L}_{\Omega}$.

(iii) Each sequent $S$ of the form $\Gamma_{0}, p, \Gamma_{1}, \cdots, \Gamma_{\lambda-1}, p, \Gamma_{\lambda} \Rightarrow A$ in GpsUL $^{*}$ has the form $\Gamma_{0}, p_{i_{1}}, \Gamma_{1}, \cdots, \Gamma_{\lambda-1}, p_{i_{\lambda}}, \Gamma_{\lambda} \Rightarrow A$ in $\mathbf{G p s}_{\mathbf{U L}_{2}}$, where $p$ does not occur in $\Gamma_{k}$ for all $0 \leqslant k \leqslant \lambda$ and, $i_{k} \neq i_{l}$ for all $1 \leqslant k<l \leqslant \lambda$. Define $v_{l}(S)=\left\{i_{1}, \cdots, i_{\lambda}\right\}, v_{r}(S)=\left\{j_{1}\right\}$ if $A$ is an eigenvariable with the identification number $j_{1}$ and, $v_{r}(S)=\varnothing$ if $A$ isn't an eigenvariable.

Let $G$ be a hypersequent of $\mathrm{GpsUL}_{\Omega}$ in the form $S_{1}|\cdots| S_{n}$ then $v_{l}\left(S_{k}\right) \cap v_{l}\left(S_{l}\right)=\varnothing$ and $v_{r}\left(S_{k}\right) \cap v_{r}\left(S_{l}\right)=\varnothing$ for all $1 \leqslant k<l \leqslant n$. Define $v_{l}(G)=\bigcup_{k=1}^{n} v_{l}\left(S_{k}\right), v_{r}(G)=\bigcup_{k=1}^{n} v_{r}\left(S_{k}\right)$.

(iv) A hypersequent $G$ of $\mathrm{GpsUL}_{\Omega}$ is called closed if $v_{l}(G)=v_{r}(G)$. Two hypersequents $G^{\prime}$ and $G^{\prime \prime}$ of $\mathrm{GpsUL}_{\Omega}$ are called disjoint if $v_{l}\left(G^{\prime}\right) \cap v_{l}\left(G^{\prime \prime}\right)=\varnothing, v_{l}\left(G^{\prime}\right) \cap v_{r}\left(G^{\prime \prime}\right)=\varnothing, v_{r}\left(G^{\prime}\right) \cap v_{l}\left(G^{\prime \prime}\right)=\varnothing$ and 
$v_{r}\left(G^{\prime}\right) \cap v_{r}\left(G^{\prime \prime}\right)=\varnothing . G^{\prime \prime}$ is a copy of $G^{\prime}$ if they are disjoint and there exist two bijections $\sigma_{l}: v_{l}\left(G^{\prime}\right) \rightarrow v_{l}\left(G^{\prime \prime}\right)$ and $\sigma_{r}: v_{r}\left(G^{\prime}\right) \rightarrow v_{r}\left(G^{\prime \prime}\right)$ such that $G^{\prime \prime}$ can be obtained by applying $\sigma_{l}$ to antecedents of sequents in $G^{\prime}$ and $\sigma_{r}$ to succedents of sequents in $G^{\prime}$.

(v) A hypersequent $G\left|G_{1}\right| G_{2}$ can be contracted as $G \mid G_{1}$ in $\mathbf{G p s U L}_{\Omega}$ under certain conditions given in Construction 3, which we called the constraint external contraction rule and denote by $\frac{G^{\prime}\left|G_{1}\right| G_{2}}{G^{\prime} \mid G_{1}}\left(E C_{\Omega}\right)$.

(vi) $(E W)$ is forbidden in $\mathbf{G p s U L}_{\Omega}$ and $(E C)$ and (CUT) are replaced with $\left(E C_{\Omega}\right)$ and $(W C T)$, respectively.

(vii) Two rules $\left(\wedge_{r}\right)$ and $\left(\vee_{l}\right)$ of $\mathbf{G L}$ are replaced with $\frac{G_{1}\left|\Gamma_{1} \Rightarrow A \quad G_{2}\right| \Gamma_{2} \Rightarrow B}{G_{1}\left|G_{2}\right| \Gamma_{1} \Rightarrow A \wedge B \mid \Gamma_{2} \Rightarrow A \wedge B}\left(\wedge_{r w}\right)$ and $\frac{G_{1}\left|\Gamma_{1}, A, \Delta_{1} \Rightarrow C_{1} \quad G_{2}\right| \Gamma_{2}, B, \Delta_{2} \Rightarrow C_{2}}{G_{1}\left|G_{2}\right| \Gamma_{1}, A \vee B, \Delta_{1} \Rightarrow C_{1} \mid \Gamma_{2}, A \vee B, \Delta_{2} \Rightarrow C_{2}}\left(\vee_{l w}\right)$ in $G_{p s U L}$, respectively.

(viii) $G_{1} \mid S_{1}$ and $G_{2} \mid S_{2}$ are closed and disjoint for each two-premise inference rule $\frac{G_{1}\left|S_{1} G_{2}\right| S_{2}}{G_{1}\left|G_{2}\right| H^{\prime}}(I I)$ of $\mathrm{GpsUL}_{\Omega}$ and, $G^{\prime} \mid S^{\prime}$ is closed for each one-premise inference rule $\frac{G^{\prime} \mid S^{\prime}}{G^{\prime} \mid S^{\prime \prime}}(I)$.

Proposition 1. Let $\frac{G^{\prime} \mid S^{\prime}}{G^{\prime} \mid S^{\prime \prime}}(I)$ and $\frac{G_{1}\left|S_{1} G_{2}\right| S_{2}}{G_{1}\left|G_{2}\right| H^{\prime}}(I I)$ be inference rules of $\mathbf{G p s U L}_{\Omega}$. Then, $v_{l}\left(G^{\prime} \mid S^{\prime \prime}\right)=$ $v_{r}\left(G^{\prime} \mid S^{\prime \prime}\right)=v_{r}\left(G^{\prime} \mid S^{\prime}\right)=v_{l}\left(G^{\prime} \mid S^{\prime}\right)$ and $v_{l}\left(G_{1}\left|G_{2}\right| H^{\prime}\right)=v_{l}\left(G_{1} \mid S_{1}\right) \cup v_{l}\left(G_{2} \mid S_{2}\right)=v_{r}\left(G_{1}\left|G_{2}\right| H^{\prime}\right)=$ $v_{r}\left(G_{1} \mid S_{1}\right) \cup v_{r}\left(G_{2} \mid S_{2}\right)$.

Proof. Although (WCT) makes t's in its premises disappear in its conclusion; it has no effect on identification numbers of the eigenvariable $p$ in a hypersequent because $t$ is a constant in $\mathbf{G p s U L}_{\Omega}$ and is distinguished from propositional variables.

Definition 6. Let $G$ be a closed hypersequent of $\mathbf{G p s U L}_{\Omega}$ and $S \in G$. [S $]_{G}:=\cap\left\{H: S \in H \subseteq G, v_{l}(H)=\right.$ $\left.v_{r}(H)\right\}$ is called a minimal closed unit of $G$.

\section{The Generalized Density Rule $(\mathcal{D})$ for $\mathrm{GpsUL}_{\Omega}$}

In this section, $\mathbf{G L}_{\Omega}^{\text {cf }}$ is $\mathbf{G}_{\mathbf{p s}} \mathbf{U L}_{\Omega}$ without $\left(E C_{\Omega}\right)$. Generally, $A, B, C, \cdots$, denote a formula other than an eigenvariable $p_{i}$.

Construction 1. Given a proof $\tau^{*}$ of $H \equiv G \mid \Gamma, p_{j}, \Delta \Rightarrow p_{j}$ in $\mathbf{G L}_{\Omega^{\text {cf }}}^{\text {cf }}$ let $T h_{\tau^{*}}\left(p_{j} \Rightarrow p_{j}\right)=\left(H_{0}, \cdots, H_{n}\right)$, where $H_{0} \equiv p_{j} \Rightarrow p_{j}, H_{n} \equiv H$. By $\Gamma_{k}, p_{j}, \Delta_{k} \Rightarrow p_{j}$, we denote the sequent containing $p_{j}$ in $H_{k}$. Then, $\Gamma_{0}=\varnothing$, $\Delta_{0}=\varnothing, \Gamma_{n}=\Gamma$ and $\Delta_{n}=\Delta$. Hypersequents $\left\langle H_{k}\right\rangle_{j}^{-},\left\langle H_{k}\right\rangle_{j}^{+}$and their proofs $\left\langle\tau^{*}\right\rangle_{j}^{-}\left(\left\langle H_{k}\right\rangle_{j}^{-}\right),\left\langle\tau^{*}\right\rangle_{j}^{+}\left(\left\langle H_{k}\right\rangle_{j}^{+}\right)$ are constructed inductively for all $0 \leqslant k \leqslant n$ in the following such that $\Gamma_{k} \Rightarrow t \in\left\langle H_{k}\right\rangle_{j}^{-}, \Delta_{k} \Rightarrow t \in\left\langle H_{k}\right\rangle_{j}^{+}$, and $\left\langle H_{k}\right\rangle_{j}^{+} \backslash\left\{\Delta_{k} \Rightarrow t\right\} \mid\left\langle H_{k}\right\rangle_{j}^{-} \backslash\left\{\Gamma_{k} \Rightarrow t\right\}=H_{k} \backslash\left\{\Gamma_{k}, p_{j}, \Delta_{k} \Rightarrow p_{j}\right\}$.

(i) $\left\langle H_{0}\right\rangle_{j}^{-}:=\left\langle H_{0}\right\rangle_{j}^{+}:=\Rightarrow t,\left\langle\tau^{*}\right\rangle_{j}^{-}\left(\left\langle H_{0}\right\rangle_{j}^{-}\right)$and $\left\langle\tau^{*}\right\rangle_{j}^{+}\left(\left\langle H_{0}\right\rangle_{j}^{+}\right)$are built up with $\Rightarrow t$.

(ii) Let $\frac{G^{\prime}\left|S^{\prime} G^{\prime \prime}\right| S^{\prime \prime}}{G^{\prime}\left|G^{\prime \prime}\right| H^{\prime}}(I I)$ (or $\frac{G^{\prime} \mid S^{\prime}}{G^{\prime} \mid S^{\prime \prime}}(I)$ ) be in $\tau^{*}, H_{k}=G^{\prime} \mid S^{\prime}$ and $H_{k+1}=G^{\prime}\left|G^{\prime \prime}\right| H^{\prime}$ (accordingly $H_{k+1}=G^{\prime} \mid S^{\prime \prime}$ for $\left.(I)\right)$ for some $0 \leqslant k \leqslant n-1$. There are three cases to be considered.

Case 1. $S^{\prime}=\Gamma_{k}, p_{j}, \Delta_{k} \Rightarrow p_{j}$. If all focus formula(s) of $S^{\prime}$ is (are) contained in $\Gamma_{k}$

$$
\begin{gathered}
\left\langle H_{k+1}\right\rangle_{j}^{-}:=\left(\left\langle H_{k}\right\rangle_{j}^{-} \backslash\left\{\Gamma_{k} \Rightarrow t\right\}\right)\left|G^{\prime \prime}\right| H^{\prime} \backslash\left\{\Gamma_{k+1}, p_{j}, \Delta_{k+1} \Rightarrow p_{j}\right\} \mid \Gamma_{k+1} \Rightarrow t \\
\left\langle H_{k+1}\right\rangle_{j}^{+}:=\left\langle H_{k}\right\rangle_{j}^{+}
\end{gathered}
$$


(accordingly $\left\langle H_{k+1}\right\rangle_{j}^{-}=\left\langle H_{k}\right\rangle_{j}^{-} \backslash\left\{\Gamma_{k} \Rightarrow t\right\} \mid \Gamma_{k+1} \Rightarrow t$ for $\left.(I)\right)$ and, $\left\langle\tau^{*}\right\rangle_{j}^{-}\left(\left\langle H_{k+1}\right\rangle_{j}^{-}\right)$is constructed by combining the derivation $\left\langle\tau^{*}\right\rangle_{j}^{-}\left(\left\langle H_{k}\right\rangle_{j}^{-}\right)$and $\frac{\left\langle H_{k}\right\rangle_{j}^{-} G^{\prime \prime} \mid S^{\prime \prime}}{\left\langle H_{k+1}\right\rangle_{j}^{-}}(I I)$ (accordingly $\frac{\left\langle H_{k}\right\rangle_{j}^{-}}{\left\langle H_{k+1}\right\rangle_{j}^{-}}(I)$ for (I)) and, $\left\langle\tau^{*}\right\rangle_{j}^{+}\left(\left\langle H_{k+1}\right\rangle_{j}^{+}\right)$is constructed by combining $\left\langle\tau^{*}\right\rangle_{j}^{+}\left(\left\langle H_{k}\right\rangle_{j}^{+}\right)$and $\frac{\left\langle H_{k}\right\rangle_{j}^{+}}{\left\langle H_{k+1}\right\rangle_{j}^{+}}\left(I D_{\Omega}\right)$. The case of all focus formula(s) of $S^{\prime}$ contained in $\Delta_{k}$ is dealt with by a procedure dual to above and omitted.

Case 2. $S^{\prime} \in\left\langle H_{k}\right\rangle_{j}^{-} .\left\langle H_{k+1}\right\rangle_{j}^{-}:=\left(\left\langle H_{k}\right\rangle_{j}^{-} \backslash\left\{S^{\prime}\right\}\right)\left|G^{\prime \prime}\right| H^{\prime}$ (accordingly $\left\langle H_{k+1}\right\rangle_{j}^{-}=\left\langle H_{k}\right\rangle_{j}^{-} \backslash\left\{S^{\prime}\right\} \mid S^{\prime \prime}$ for $(I)$ ), $\left\langle H_{k+1}\right\rangle_{j}^{+}:=\left\langle H_{k}\right\rangle_{j}^{+}$and $\left\langle\tau^{*}\right\rangle_{j}^{-}\left(\left\langle H_{k+1}\right\rangle_{j}^{-}\right)$is constructed by combining the derivation $\left\langle\tau^{*}\right\rangle_{j}^{-}\left(\left\langle H_{k}\right\rangle_{j}^{-}\right)$and $\frac{\left\langle H_{k}\right\rangle_{j}^{-} G^{\prime \prime} \mid S^{\prime \prime}}{\left\langle H_{k+1}\right\rangle_{j}^{-}}(I I)$ (accordingly $\frac{\left\langle H_{k}\right\rangle_{j}^{-}}{\left\langle H_{k+1}\right\rangle_{j}^{-}}(I)$ for $\left.(I)\right)$ and,$\left\langle\tau^{*}\right\rangle_{j}^{+}\left(\left\langle H_{k+1}\right\rangle_{j}^{+}\right)$is constructed by combining $\left\langle\tau^{*}\right\rangle_{j}^{+}\left(\left\langle H_{k}\right\rangle_{j}^{+}\right)$and $\frac{\left\langle H_{k}\right\rangle_{j}^{+}}{\left\langle H_{k+1}\right\rangle_{j}^{+}}\left(I D_{\Omega}\right)$.

Case 3. $S^{\prime} \in\left\langle H_{k}\right\rangle_{j}^{+}$. It is dealt with by a procedure dual to Case 2 and omitted.

Definition 7. The manipulation described in Construction 1 is called the derivation-splitting operation when it is applied to a derivation and the splitting operation when applied to a hypersequent.

Corollary 1. Let $\vdash_{\mathrm{GL}_{\Omega}^{\text {cf }}} G \mid \Gamma, p_{1}, \Delta \Rightarrow p_{1}$. Then, there exist two hypersequents $G_{1}$ and $G_{2}$ such that $G=$ $G_{1} \cup G_{2}, G_{1} \cap G_{2}=\varnothing, \vdash_{\mathbf{G L}_{\Omega}^{\mathrm{cf}}} G_{1} \mid \Gamma \Rightarrow t$ and $\vdash_{\mathbf{G L}_{\Omega}^{\mathrm{cf}}} G_{2} \mid \Delta \Rightarrow t$.

Construction 2. Given a proof $\tau^{*}$ of $H \equiv G\left|\Pi \Rightarrow p_{j}\right| \Gamma, p_{j}, \Delta \Rightarrow A$ in $\mathbf{G L}_{\Omega^{\prime}}^{\text {cf }}$ let $T h_{\tau^{*}}\left(p_{j} \Rightarrow p_{j}\right)=\left(H_{0}, \cdots, H_{n}\right)$, where $H_{0} \equiv p_{j} \Rightarrow p_{j}$ and $H_{n} \equiv H$. Then, there exists $1 \leqslant m \leqslant n$ such that $H_{m}$ is in the form $G^{\prime} \mid \Pi^{\prime} \Rightarrow$ $p_{j} \mid \Gamma^{\prime}, p_{j}, \Delta^{\prime} \Rightarrow A^{\prime}$ and $H_{m-1}$ is in the form $G^{\prime \prime} \mid \Gamma^{\prime \prime}, p_{j}, \Delta^{\prime \prime} \Rightarrow p_{j}$. A proof of $G \mid \Gamma, \Pi, \Delta \Rightarrow A$ in $\mathbf{G L}_{\Omega}^{\text {cf }}$ is constructed by induction on $n-m$ as follows:

- For the base step, let $n-m=0$. Then, $\frac{H_{n-1} \equiv G^{\prime}\left|\Pi^{\prime}, \Gamma^{\prime}, p_{j}, \Delta^{\prime}, \Pi^{\prime \prime \prime} \Rightarrow p_{j} \quad G^{\prime \prime}\right| \Gamma^{\prime \prime}, \Pi^{\prime \prime}, \Delta^{\prime \prime} \Rightarrow A}{H_{n} \equiv G^{\prime}\left|G^{\prime \prime}\right| \Pi^{\prime}, \Pi^{\prime \prime}, \Pi^{\prime \prime \prime} \Rightarrow p_{j} \mid \Gamma^{\prime \prime}, \Gamma^{\prime}, p_{j}, \Delta^{\prime}, \Delta^{\prime \prime} \Rightarrow A}(C O M) \in \tau^{*}$, where $G^{\prime} \mid G^{\prime \prime}=G$ and $\Pi^{\prime}, \Pi^{\prime \prime}, \Pi^{\prime \prime \prime}=\Pi$ and $\Gamma^{\prime \prime}, \Gamma^{\prime}=\Gamma$ and $\Delta^{\prime}, \Delta^{\prime \prime}=\Delta$. It follows from Corollary 1 that there exist $G_{1}^{\prime}$ and $G_{2}^{\prime}$ such that $G^{\prime}=G_{1}^{\prime} \cup G_{2}^{\prime}, G_{1}^{\prime} \cap G_{2}^{\prime}=\varnothing, \vdash_{\mathbf{G L}_{\Omega}^{\text {cf }}} G_{1}^{\prime} \mid \Pi^{\prime}, \Gamma^{\prime} \Rightarrow t$ and $\vdash_{\mathrm{GL}_{\Omega}^{\mathrm{cf}}} G_{2}^{\prime} \mid \Delta^{\prime}, \Pi^{\prime \prime \prime} \Rightarrow t$. Then, $G \mid \Gamma, \Pi, \Delta \Rightarrow A$ is proved as follows:

\begin{tabular}{|c|c|c|}
\hline$\frac{G^{\prime \prime} \mid \Gamma^{\prime \prime}, \Pi^{\prime \prime}, \Delta^{\prime \prime} \Rightarrow A}{G^{\prime \prime} \mid \Gamma^{\prime \prime}, t, \Pi^{\prime \prime}, \Delta^{\prime \prime} \Rightarrow A}\left(t_{l}\right)$ & $\frac{G_{1}^{\prime} \mid \Pi^{\prime}, \Gamma^{\prime} \Rightarrow t}{G_{1}^{\prime} \mid \Gamma^{\prime}, \Pi^{\prime} \Rightarrow t}(W C M)$ & \\
\hline \multicolumn{2}{|c|}{$\begin{array}{c}G^{\prime \prime}\left|G_{1}^{\prime}\right| \Gamma^{\prime \prime}, \Gamma^{\prime}, \Pi^{\prime}, \Pi^{\prime \prime}, \Delta^{\prime \prime} \Rightarrow A \\
G^{\prime \prime}\left|G_{1}^{\prime}\right| \Gamma^{\prime \prime}, \Gamma^{\prime}, \Pi^{\prime}, \Pi^{\prime \prime}, t, \Delta^{\prime \prime} \Rightarrow A\end{array}\left(t_{l}\right)$} & $\frac{G_{2}^{\prime} \mid \Delta^{\prime}, \Pi^{\prime \prime \prime} \Rightarrow t}{G^{\prime} \mid \Pi^{\prime \prime \prime} \Delta^{\prime} \Rightarrow t}(W C M)$ \\
\hline \multicolumn{3}{|c|}{$G^{\prime \prime}\left|G_{1}^{\prime}\right| G_{2}^{\prime} \mid \Gamma^{\prime \prime}, \Gamma^{\prime}, \Pi^{\prime}, \Pi^{\prime \prime}, \Pi^{\prime \prime \prime}, \Delta^{\prime}, \Delta^{\prime \prime} \Rightarrow A$} \\
\hline
\end{tabular}

- For the induction step, let $n-m>0$. Then, it is treated using applications of the induction hypothesis to the premise followed by an application of the relevant rule. For example, let $\frac{H_{n-1}=G^{\prime}\left|\Pi \Rightarrow p_{j}\right| \Sigma^{\prime}, \Gamma^{\prime \prime}, p_{j}, \Delta^{\prime \prime}, \Sigma^{\prime \prime \prime} \Rightarrow A^{\prime} \quad G^{\prime \prime} \mid \Gamma^{\prime}, \Sigma^{\prime \prime}, \Delta^{\prime} \Rightarrow A}{H_{n}=G^{\prime}\left|\Pi \Rightarrow p_{j}\right| \Sigma^{\prime}, \Sigma^{\prime \prime}, \Sigma^{\prime \prime \prime} \Rightarrow A^{\prime}\left|G^{\prime \prime}\right| \Gamma^{\prime}, \Gamma^{\prime \prime}, p_{j}, \Delta^{\prime \prime}, \Delta^{\prime} \Rightarrow A}(C O M) \in \tau^{*}$, where $G^{\prime}\left|G^{\prime \prime}\right| \Sigma^{\prime}, \Sigma^{\prime \prime}, \Sigma^{\prime \prime \prime} \Rightarrow A^{\prime}=G$ and $\Gamma^{\prime}, \Gamma^{\prime \prime}=\Gamma$ and $\Delta^{\prime \prime}, \Delta^{\prime}=\Delta$. By the induction hypothesis, we obtain a derivation of $G \mid \Gamma, \Pi, \Delta \Rightarrow A$ :

$$
\frac{G^{\prime}\left|\Sigma^{\prime}, \Gamma^{\prime \prime}, \Pi, \Delta^{\prime \prime}, \Sigma^{\prime \prime \prime} \Rightarrow A^{\prime} \quad G^{\prime \prime}\right| \Gamma^{\prime}, \Sigma^{\prime \prime}, \Delta^{\prime} \Rightarrow A}{G^{\prime}\left|\Sigma^{\prime}, \Sigma^{\prime \prime}, \Sigma^{\prime \prime \prime} \Rightarrow A^{\prime}\right| G^{\prime \prime} \mid \Gamma^{\prime}, \Gamma^{\prime \prime}, \Pi, \Delta^{\prime \prime}, \Delta^{\prime} \Rightarrow A}(C O M) .
$$


Definition 8. The manipulation described in Construction 2 is called the derivation-splicing operation when it is applied to a derivation and the splicing operation when applied to a hypersequent.

Corollary 2. If $\vdash_{\mathrm{GL}_{\Omega}^{\mathrm{cf}}} G\left|\Pi \Rightarrow p_{j}\right| \Gamma, p_{j}, \Delta \Rightarrow A$, then $\vdash_{\mathrm{GL}_{\Omega}^{\mathrm{cf}}} G \mid \Gamma, \Pi, \Delta \Rightarrow A$.

Definition 9. (i) Let $\vdash_{\mathbf{G L}_{\Omega}^{\text {cf }}} H \equiv G \mid \Gamma, p_{j}, \Delta \Rightarrow p_{j}$. Define $\langle H\rangle_{j}^{-}=G_{1}\left|\Gamma \Rightarrow t,\langle H\rangle_{j}^{+}=G_{2}\right| \Delta \Rightarrow t$ and $D_{j}(H)=\left\{G_{1}\left|\Gamma \Rightarrow t, G_{2}\right| \Delta \stackrel{\Omega}{\Rightarrow} t\right\}$, where $G_{1}$ and $G_{2}$ are determined by Corollary 1.

(ii) Let $\vdash \mathrm{GL}_{\Omega}^{\text {cf }} H \equiv G\left|\Pi \Rightarrow p_{j}\right| \Gamma, p_{j}, \Delta \Rightarrow A$. Define $D_{j}(H)=\{G \mid \Gamma, \Pi, \Delta \Rightarrow A\}=\langle H\rangle_{j}$.

(iii) Let $\vdash_{\mathbf{G L}_{\Omega}^{\mathrm{cf}}} G$. $D_{j}(G)=\{G\}$ if $p_{j}$ does not occur in $G$.

(iv) Let $\vdash_{\mathbf{G L}_{\Omega}^{\mathrm{cf}}} G_{i}$ for all $1 \leqslant i \leqslant n$. Define $D_{j}\left(\left\{G_{1}, \cdots, G_{n}\right\}\right)=D_{j}\left(G_{1}\right) \cup \cdots \cup D_{j}\left(G_{n}\right)$.

(v) Let $\vdash_{G_{L}}^{\text {cf }} G$ and $K=\{1, \cdots, n\} \subseteq v(G)$. Define $D_{K}(G)=D_{n}\left(\cdots D_{2}\left(D_{1}(G)\right) \cdots\right)$. Especially, define $\mathcal{D}(G)=D_{v_{l}(G)}(\stackrel{\Omega}{G})$.

Theorem 3. Let $\vdash_{\mathbf{G L}_{\Omega}^{\text {cf }}} G$. Then, $\vdash_{\mathbf{G L}_{\Omega}^{\text {cf }}} H$ for all $H \in \mathcal{D}(G)$.

Proof. Immediately from Corollaries 1, 2 and Definition 9.

Lemma 2. Let $G^{\prime}$ be a minimal closed unit of $G \mid G^{\prime}$. Then, $G^{\prime}$ has the form $\Gamma \Rightarrow A\left|\Gamma_{i_{2}} \Rightarrow p_{i_{2}}\right| \cdots \mid \Gamma_{i_{n}} \Rightarrow p_{i_{n}}$ if there exists one sequent $\Gamma \Rightarrow A \in G^{\prime}$ such that $A$ is not an eigenvariable otherwise $G^{\prime}$ has the form $\Gamma_{i_{1}} \Rightarrow p_{i_{1}}|\cdots| \Gamma_{i_{n}} \Rightarrow p_{i_{n}}$.

Proof. Define $G_{1}=\Gamma \Rightarrow A$ in Construction 5.2 in [10]. Then, $\varnothing=v_{r}\left(G_{1}\right) \subseteq v_{l}\left(G_{1}\right)$. Suppose that $G_{k}$ is constructed such that $v_{r}\left(G_{k}\right) \subseteq v_{l}\left(G_{k}\right)$. If $v_{l}\left(G_{k}\right)=v_{r}\left(G_{k}\right)$, the procedure terminates and $n:=k$; otherwise, $v_{l}\left(G_{k}\right) \backslash v_{r}\left(G_{k}\right) \neq \varnothing$ and define $i_{k+1}$ to be an identification number in $v_{l}\left(G_{k}\right) \backslash v_{r}\left(G_{k}\right)$. Then, there exists $\Gamma_{i_{k+1}} \Rightarrow p_{i_{k+1}} \in G \backslash G_{k}$ by $v_{l}(G)=v_{r}(G)$ and define $G_{k+1}=G_{k} \mid \Gamma_{i_{k+1}} \Rightarrow p_{i_{k+1}}$. Thus, $v_{r}\left(G_{k+1}\right)=v_{r}\left(G_{k}\right) \cup\left\{i_{k+1}\right\} \subseteq v_{l}\left(G_{k}\right) \subseteq v_{l}\left(G_{k+1}\right)$. Hence, there exists a sequence $i_{2}, \cdots, i_{n}$ of identification numberssuch that $v_{r}\left(G_{k}\right) \subseteq v_{l}\left(G_{k}\right)$ for all $1 \leqslant k \leqslant n$, where $G_{1}=\Gamma \Rightarrow A, G_{k}=\Gamma \Rightarrow A\left|\Gamma_{i_{2}} \Rightarrow p_{i_{2}}\right| \cdots \mid \Gamma_{i_{k}} \Rightarrow p_{i_{k}}$ for all $2 \leqslant k \leqslant n$. Therefore, $G^{\prime}$ has the form $\Gamma \Rightarrow A\left|\Gamma_{i_{2}} \Rightarrow p_{i_{2}}\right| \cdots \mid \Gamma_{i_{n}} \Rightarrow p_{i_{n}}$.

Definition 10. Let $G^{\prime}$ be a minimal closed unit of $G \mid G^{\prime} . G^{\prime}$ is a splicing unit if it has the form $\Gamma \Rightarrow A \mid \Gamma_{i_{2}} \Rightarrow$ $p_{i_{2}}|\cdots| \Gamma_{i_{n}} \Rightarrow p_{i_{n}}$. G' is a splitting unit if it has the form $\Gamma_{i_{1}} \Rightarrow p_{i_{1}}|\cdots| \Gamma_{i_{n}} \Rightarrow p_{i_{n}}$.

Lemma 3. Let $G^{\prime}$ be a splicing unit of $G \mid G^{\prime}$ in the form $\Gamma \Rightarrow A\left|\Gamma_{i_{2}} \Rightarrow p_{i_{2}}\right| \cdots \mid \Gamma_{i_{n}} \Rightarrow p_{i_{n}}$ and $K=\left\{i_{2}, \cdots, i_{n}\right\}$. Then, $\left|D_{K}\left(G \mid G^{\prime}\right)\right|=1$.

Proof. By the construction in the proof of Lemma $2, i_{k} \in v_{l}\left(G_{k-1}\right)$ for all $2 \leqslant k \leqslant n$. Then, $p_{i_{2}} \in \Gamma$ and $D_{i_{2}}\left(G \mid G^{\prime}\right)=G\left|\Gamma\left[\Gamma_{i_{2}}\right] \Rightarrow A\right| \Gamma_{i_{3}} \Rightarrow p_{i_{3}}|\cdots| \Gamma_{i_{n}} \Rightarrow p_{i_{n}}$, where $\Gamma\left[\Gamma_{i_{2}}\right]$ is obtained by replacing $p_{i_{2}}$ in $\Gamma$ with $\Gamma_{i_{2}}$. Then, $p_{i_{3}} \in \Gamma\left[\Gamma_{i_{2}}\right]$. Repeatedly, we get $D_{i_{2} \cdots i_{n}}\left(G \mid G^{\prime}\right)=D_{K}\left(G \mid G^{\prime}\right)=G \mid \Gamma\left[\Gamma_{i_{2}}\right] \cdots\left[\Gamma_{i_{n}}\right] \Rightarrow A$.

This shows that $D_{K}\left(G \mid G^{\prime}\right)$ is constructed by repeatedly applying splicing operations.

Definition 11. Let $G^{\prime}$ be a minimal closed unit of $G \mid G^{\prime}$. Define $V_{G^{\prime}}=v\left(G^{\prime}\right), E_{G^{\prime}}=\left\{(i, j) \mid \Gamma, p_{i}, \Delta \Rightarrow p_{j} \in G^{\prime}\right\}$ and, $j$ is called the child node of $i$ for all $(i, j) \in E_{G^{\prime}}$. We call $\Omega_{G^{\prime}}=\left(V_{G^{\prime}}, E_{G^{\prime}}\right)$ the $\Omega$-graph of $G^{\prime}$.

Let $G^{\prime}$ be a splitting unit of $G \mid G^{\prime}$ in the form $\Gamma_{1} \Rightarrow p_{1}|\cdots| \Gamma_{n} \Rightarrow p_{n}$. Then, each node of $\Omega_{G^{\prime}}$ has one and only one child node. Thus, there exists one cycle in $\Omega_{G^{\prime}}$ by $\left|V_{G^{\prime}}\right|=n<\infty$. Assume that, without loss of generality, $(1,2),(2,3), \cdots,(i, 1)$ is the cycle of $\Omega_{G^{\prime}}$. Then, $p_{1} \in \Gamma_{2}, p_{2} \in \Gamma_{3}, \cdots, p_{i-1} \in \Gamma_{i}$ and $p_{i} \in \Gamma_{1}$. Thus, $D_{i \cdots 2}\left(G \mid G^{\prime}\right)=G \mid \Gamma_{1}\left[\Gamma_{i}\right]\left[\Gamma_{i-1}\right] \cdots\left[\Gamma_{2}\right] \Rightarrow p_{1}$ is in the form $G \mid \Gamma^{\prime}, p_{1}, \Delta^{\prime} \Rightarrow p_{1}$. By a suitable permutation $\sigma$ of $i+1, \cdots, n$, we get $D_{i \cdots 2 \sigma(i+1 \cdots n)}\left(G \mid G^{\prime}\right)=G \mid \Gamma_{1}\left[\Gamma_{i}\right]\left[\Gamma_{i-1}\right] \cdots\left[\Gamma_{2}\right]\left[\Gamma_{\sigma(i+1)}\right] \cdots\left[\Gamma_{\sigma(n)}\right] \Rightarrow p_{1}=$ $G \mid \Gamma, p_{1}, \Delta \Rightarrow p_{1}$. This process also shows that there exists only one cycle in $\Omega_{G^{\prime}}$. Then, we introduce the following definition. 
Definition 12. (i) $\Gamma_{j} \Rightarrow p_{j}$ is called a splitting sequent of $G^{\prime}$ and $p_{j}$ its corresponding splitting variable for all $1 \leqslant j \leqslant i$.

(ii) Let $K=\{1,2, \cdots, n\}$ and $D_{1}\left(G \mid \Gamma, p_{1}, \Delta \Rightarrow p_{1}\right)=\left\{G_{1}\left|\Gamma \Rightarrow t, G_{2}\right| \Delta \Rightarrow t\right\}$. Define $\left\langle G \mid G^{\prime}\right\rangle_{K}^{-}=G_{1} \mid \Gamma \Rightarrow t$, $\left\langle G \mid G^{\prime}\right\rangle_{K}^{+}=G_{2} \mid \Delta \Rightarrow$ tand $D_{K}\left(G \mid G^{\prime}\right)=\left\{\left\langle G \mid G^{\prime}\right\rangle_{K}^{+},\left\langle G \mid G^{\prime}\right\rangle_{K}^{-}\right\}$.

Lemma 4. If $G^{\prime}$ be a splitting unit of $G \mid G^{\prime}, K=v\left(G^{\prime}\right)$ and $k$ be a splitting variable of $G^{\prime}$. Then, $D_{K \backslash\{k\}}\left(G \mid G^{\prime}\right)$ is constructed by repeatedly applying splicing operations and only the last operation $D_{k}$ is a splitting operation.

Construction 3 (The constrained external contraction rule). Let $H \equiv G^{\prime}\left|\left\{[S]_{H}\right\}_{1}\right|\left\{[S]_{H}\right\}_{2^{\prime}}\left\{[S]_{H}\right\}_{1}$ and $\left\{[S]_{H}\right\}_{2}$ be two copies of a minimal closed unit $[S]_{H}$, where we put two copies into \{\}$_{1}$ and \{\}$_{2}$ in order to distinguish them. For any splitting unit $\left[S^{\prime}\right]_{H} \subseteq G^{\prime},\left\{[S]_{H}\right\}_{1} \mid\left\{[S]_{H}\right\}_{2} \subseteq\langle H\rangle_{K}^{-}$or $\left\{[S]_{H}\right\}_{1} \mid\left\{[S]_{H}\right\}_{2} \subseteq\langle H\rangle_{K^{\prime}}^{+}$ where $K=v\left(\left[S^{\prime}\right]_{H}\right)$. Then, $G^{\prime \prime} \mid\left\{[S]_{H}\right\}_{1}$ is constructed by cutting off $\left\{[S]_{H}\right\}_{2}$ and some sequents in $G^{\prime}$ as follows.

(i) If $\left\{[S]_{H}\right\}_{1}$ and $\left\{[S]_{H}\right\}_{2}$ are two splicing units, then $G^{\prime \prime}:=G^{\prime}$;

(ii) If $\left\{[S]_{H}\right\}_{1}$ and $\left\{[S]_{H}\right\}_{2}$ are two splitting units and, $k, k^{\prime}$ their splitting variables, respectively, $K=v\left(\left\{[S]_{H}\right\}_{1}\right), K^{\prime}=v\left(\left\{[S]_{H}\right\}_{2}\right), D_{K \backslash\{k\}}\left(\left\{[S]_{H}\right\}_{1}\right)=\Gamma, p_{k}, \Delta \Rightarrow p_{k}, D_{K^{\prime} \backslash\left\{k^{\prime}\right\}}\left(\left\{[S]_{H}\right\}_{2}\right)=\Gamma, p_{k^{\prime}}, \Delta \Rightarrow p_{k^{\prime}}$, $D_{K \cup K^{\prime}}(H)=\left\{G_{1}^{\prime}|\Gamma \Rightarrow t| \Gamma \Rightarrow t, G_{2}^{\prime}\left|\Delta \Rightarrow t, G_{2}^{\prime \prime}\right| \Delta \Rightarrow t\right\}$ or $D_{K \cup K^{\prime}}(H)=\left\{G_{1}^{\prime}|\Delta \Rightarrow t| \Delta \Rightarrow t, G_{2}^{\prime}\left|\Gamma \Rightarrow t, G_{2}^{\prime \prime}\right| \Gamma \Rightarrow\right.$ $t\}$, where $G_{1}^{\prime} \cup G_{2}^{\prime} \cup G_{2}^{\prime \prime}=G^{\prime}$ and $G_{2}^{\prime \prime}$ is a copy of $G_{2}^{\prime}$. Then, $G^{\prime \prime}:=G^{\prime} \backslash G_{2}^{\prime \prime}$.

The above operation is called the constrained external contraction rule, denoted by $\left\langle E C_{\Omega}^{*}\right\rangle$ and written as $\frac{G^{\prime}\left|\left\{[S]_{H}\right\}_{1}\right|\left\{[S]_{H}\right\}_{2}}{G^{\prime \prime} \mid\left\{[S]_{H}\right\}_{1}}\left\langle E C_{\Omega}^{*}\right\rangle$.

Lemma 5. If $\vdash_{\mathrm{GL}_{\Omega}^{\mathrm{cf}}} H$ as above, then $\vdash_{\mathrm{GpsuL}_{\Omega}} H^{\prime}$ for all $H^{\prime} \in \mathcal{D}\left(G^{\prime \prime} \mid\left\{[S]_{H}\right\}_{1}\right)$.

\section{Density Elimination for GpsUL ${ }^{*}$}

In this section, we adapt the separation algorithm of branches in [10] to $\mathrm{GpsUL}^{*}$ and prove the following theorem.

Theorem 4. Density elimination holds for GpsUL*

The proof of Theorem 4 runs as follows. It is sufficient to prove that the following strong density rule

$$
\frac{G_{0} \equiv G^{\prime}\left|\left\{\Gamma_{i}, p, \Delta_{i} \Rightarrow A_{i}\right\}_{i=1 \cdots n}\right|\left\{\Pi_{j} \Rightarrow p\right\}_{j=1 \cdots m}}{\mathcal{D}_{0}\left(G_{0}\right) \equiv G^{\prime} \mid\left\{\Gamma_{i}, \Pi_{j}, \Delta_{i} \Rightarrow A_{i}\right\}_{i=1 \cdots n ; j=1 \cdots m}}\left(\mathcal{D}_{0}\right)
$$

is admissible in $\mathrm{GpsUL}^{*}$, where $n, m \geqslant 1, p$ does not occur in $G^{\prime}, \Gamma_{i}, \Delta_{i}, A_{i}, \Pi_{j}$ for all $1 \leqslant i \leqslant n, 1 \leqslant j \leqslant m$.

Let $\tau$ be a proof of $G_{0}$ in GpsUL ${ }^{* *}$ by Theorem 2. Starting with $\tau$, we construct a proof $\tau^{*}$ of $G \mid G^{*}$ in $\mathbf{G L}_{\Omega}^{\text {cf }}$ by a preprocessing of $\tau$ described in Section 4 in [10].

In Step 1 of preprocessing of $\tau$, a proof $\tau^{\prime}$ is constructed by replacing inductively all applications of $\left(\wedge_{r}\right)$ and $\left(\vee_{l}\right)$ in $\tau$ with $\left(\wedge_{r w}\right)$ and $\left(\vee_{l w}\right)$ followed by an application of $(E C)$, respectively. In Step 2, a proof $\tau^{\prime \prime}$ is constructed by converting all $\frac{G_{i}^{\prime \prime \prime} \mid\left\{S_{i}^{c}\right\}^{m_{i}^{\prime}}}{G_{i}^{\prime \prime \prime} \mid S_{i}^{c}}\left(E C^{*}\right) \in \tau^{\prime}$ into $\frac{G_{i}^{\prime \prime} \mid\left\{S_{i}^{c}\right\}^{m_{i}^{\prime}}}{G_{i}^{\prime \prime} \mid\left\{S_{i}^{c}\right\}_{i}^{m_{i}^{\prime}}}\left(I D_{\Omega}\right)$, where $G_{i}^{\prime \prime \prime} \subseteq G_{i}^{\prime \prime}$. In Step 3, a proof $\tau^{\prime \prime \prime}$ is constructed by converting $\frac{G^{\prime}}{G^{\prime} \mid S^{\prime}}(E W) \in \tau^{\prime \prime}$ into $\frac{G^{\prime \prime}}{G^{\prime \prime}}\left(I D_{\Omega}\right)$, where $G^{\prime \prime} \subseteq G^{\prime}$. In Step 4, a proof $\tau^{\prime \prime \prime \prime \prime}$ is constructed by replacing some $G^{\prime} \mid \Gamma^{\prime}, p, \Delta^{\prime} \Rightarrow A^{\prime} \in \tau^{\prime \prime \prime}$ (or $G^{\prime} \mid \Gamma^{\prime} \Rightarrow p \in \tau^{\prime \prime \prime}$ ) with $G^{\prime} \mid \Gamma^{\prime}, T, \Delta^{\prime} \Rightarrow A^{\prime}$ (or $G^{\prime} \mid \Gamma^{\prime} \Rightarrow \perp$ ). In Step 5 , a proof $\tau^{*}$ is constructed by assigning the unique identification number to each occurrence of $p$ in $\tau^{\prime \prime \prime \prime}$. Let $H_{i}^{c}=G_{i}^{\prime} \mid\left\{S_{i}^{c}\right\}^{m_{i}}$ denote the unique node of $\tau^{*}$ such that $H_{i}^{c} \leqslant G_{i}^{\prime \prime} \mid\left\{S_{i}^{c}\right\}^{m_{i}}$ and $S_{i}^{c}$ is the focus sequent of $H_{i}^{c}$ in $\tau^{*}$. We call $H_{i}^{c}$, $S_{i}^{c}$ the $i$-th ( $\left.p E C\right)$-node of $\tau^{*}$ and $(p E C)$-sequent, respectively. If we ignore the replacements from Step 4, each sequent of $G$ is a copy of some sequent of $G_{0}$ and each sequent of $G^{*}$ is a copy of some contraction sequent in $\tau^{\prime}$. 


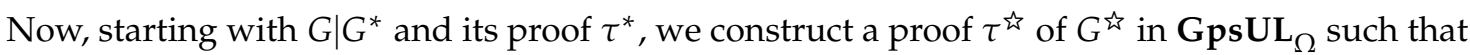
each sequent of $G^{\text {tr }}$ is a copy of some sequent of $G$. Then, $\vdash \mathrm{GpsUL}_{\Omega} \mathcal{D}\left(G^{\text {tr }}\right)$ by Theorem 3 and Lemma 5 . Then, $\vdash_{\mathrm{GpsuL}^{*}} \mathcal{D}_{0}\left(G_{0}\right)$ by Lemma 9.1 in [10].

In [10], $G^{\text {tr }}$ is constructed by eliminating $(p E C)$-sequents in $G \mid G^{*}$ one by one. In order to control the process, we introduce the set $I=\left\{H_{i_{1}}^{c}, \cdots, H_{i_{m}}^{c}\right\}$ of maximal $(p E C)$-nodes of $\tau^{*}$ (see Definition 13)

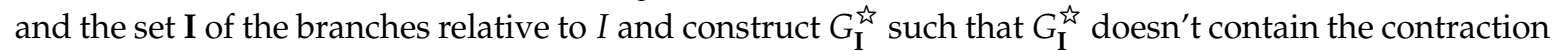
sequents lower than any node in $I$, i.e., $S_{j}^{c} \in G_{I}^{\text {in }}$ implies $H_{j}^{c} \| H_{i}^{c}$ for all $H_{i}^{c} \in I$. The procedure is called the separation algorithm of branches in [10].

The problem we encounter in $\mathrm{GpsUL}_{\Omega}$ is that Lemma 7.11 of [10] doesn't hold because new derivation-splitting operations make the conclusion of $(\mathcal{D})$-rule to be a set of hypersequents rather than one hypersequent. Then, $G_{\ddagger}^{m_{q^{\prime}}}$ generally can't be contracted to $G_{\ddagger}$ in Step 2 of Stage 1 in the

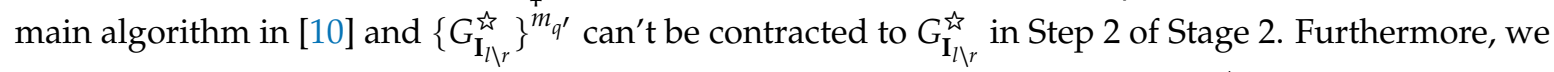
sometimes can't construct some branches to $I$ in $\mathrm{GpsUL}_{\Omega}$ before we construct $\tau_{I}^{\text {is }}$. Therefore, we have to introduce a new induction strategy for $\mathrm{GpsUL}_{\Omega}$ and don't perform the induction on the number of branches. First, we give some primary definitions and lemmas.

Definition 13. $A(p E C)$-node $H_{i}^{c}$ is maximal if no other ( $\left.p E C\right)$-node is higher than $H_{i}^{c}$. Define $I_{0}$ to be the set of maximal ( $p E C)$-nodes in $\tau^{*}$. A nonempty subset $I$ of $I_{0}$ is complete if I contains all maximal ( $\left.p E C\right)$-nodes higher than or equal to the intersection node $H_{I}^{V}$ of $I$. Define $H_{I}^{V}=H_{i}^{c}$ if $I=\left\{H_{i}^{c}\right\}$, i.e., the intersection node of a single node is itself.

Proposition 2. (i) $H_{i}^{c} \| H_{j}^{c}$ for all $i \neq j, H_{i}^{c}, H_{j}^{c} \in I_{0}$.

(ii) Let I be complete and $H_{j}^{c} \geqslant H_{I}^{V}$. Then, $H_{j}^{c} \leqslant H_{i}^{c}$ for some $H_{i}^{c} \in I$.

(iii) $I_{0}$ is complete and $\left\{H_{i}^{c}\right\}$ is complete for all $H_{i}^{c} \in I_{0}$.

(iv) If $I \subseteq I_{0}$ is complete and $|I|>1$, then $I_{l}$ and $I_{r}$ are complete, where $I_{l}$ and $I_{r}$ denote the sets of all maximal $(p E C)$-nodes in the left subtree and right subtree of $\tau^{*}\left(H_{I}^{V}\right)$, respectively.

(v) If $I_{1}, I_{2} \subseteq I_{0}$ are complete, then $I_{1} \subseteq I_{2}, I_{2} \subseteq I_{1}$ or $I_{1} \cap I_{2}=\varnothing$.

Proof. Only (v) is proved as follows. $I_{1} \subseteq I_{2}, I_{2} \subseteq I_{1}$ or $I_{1} \cap I_{2}=\varnothing$ holds by $H_{I_{2}}^{V} \leqslant H_{I_{1}}^{V}, H_{I_{1}}^{V} \leqslant H_{I_{2}}^{V}$ or $H_{I_{2}}^{V} \| H_{I_{1}}^{V}$, respectively.

Definition 14. A labeled binary tree $\rho$ is constructed inductively by the following operations:

(i) The root of $\rho$ is labeled by $I_{0}$ and leaves labeled $\left\{H_{i}^{c}\right\} \subseteq I_{0}$.

(ii) If an inner node is labeled by $I$, then its parent nodes are labeled by $I_{l}$ and $I_{r}$, where $I_{l}$ and $I_{r}$ are defined in Proposition 2(iv).

Definition 15. We define the height $o(I)$ of $I \in \rho$ by letting $o(I)=1$ for each leave $I \in \rho$ and, $o(I)=$ $\max \left\{o\left(I_{l}\right), o\left(I_{r}\right)\right\}+1$ for any non-leaf node.

Note that in Lemma 7.11 in [10] only uniqueness of $G_{H_{1}: G_{2}}^{\mathfrak{i}(J)} \mid \widehat{S_{2}}$ in $G_{H_{i_{k}}^{c}}^{i \vec{j}}$ doesn't hold in $\mathbf{G p s U L}_{\Omega}$ and the following lemma holds in $\mathrm{GpsUL}_{\Omega}$.

Lemma 6. Let $\frac{G_{1}\left|S_{1} \quad G_{2}\right| S_{2}}{H_{1} \equiv G_{1}\left|G_{2}\right| H^{\prime \prime}}(I I) \in \tau^{*}, \tau_{G_{b} \mid S_{j}^{c}}^{*} \in \tau_{H_{i}^{c}}^{i,} \frac{G_{b}\left|\left\langle G_{1} \mid S_{1}\right\rangle_{S_{j}^{c}} G_{2}\right| S_{2}}{H_{2} \equiv G_{b}\left|\left\langle G_{1}\right\rangle_{S_{j}^{c}}\right| G_{2} \mid H^{\prime \prime}}(I I) \in \tau_{G_{b} \mid S_{j}^{c}}^{*}$. Then, $H^{\prime \prime}$ is separable in $\tau_{H_{i}^{c}}^{i(J)}$ and there are some copies of $G_{H_{1}}^{i n}: G_{2} \mid \widehat{S_{2}}$ in $G_{H_{i}^{c}}^{i \vec{c}}$.

Lemma 7. (New main algorithm for $\mathrm{GpsUL}_{\Omega}$ ) Let I be a complete subset of $I_{0}$ and $\bar{I}=\left\{H_{i}^{c}: H_{i}^{c} \leqslant\right.$ $H_{j}^{c}$ for some $\left.H_{j}^{c} \in I\right\}$. Then, there exists one close hypersequent $G_{I}^{\boldsymbol{i}^{3}} \subseteq_{c} G \mid G^{*}$ and its derivation $\tau_{I}^{\hat{\imath}^{i}}$ in $\mathrm{GpsUL}_{\Omega}$ such that 
(i) $\tau_{I}^{i 3}$ is constructed by initial hypersequent $\overline{\overline{G \mid G^{*}}}\left\langle\tau^{*}\right\rangle$, the fully constraint contraction rules of the form $\frac{G_{2}}{G_{1}}\left\langle E C_{\Omega}^{*}\right\rangle$ and elimination rule of the form

$$
\left.\frac{G_{b_{1}}\left|S_{j_{1}}^{c} \quad G_{b_{2}}\right| S_{j_{2}}^{c} \cdots G_{b_{w}} \mid S_{j_{w}}^{c}}{G_{\mathbf{I}_{\mathbf{j}}}^{*}=\left\{G_{b_{k}}\right\}_{k=1}^{w} \mid G_{\mathcal{I}_{\mathbf{j}}}^{*}} \mid \tau_{\mathbf{I}_{\mathbf{j}}}^{*}\right\},
$$

where $1 \leqslant w \leqslant|I|, H_{j_{k}}^{c} \leftrightarrow H_{j_{l}}^{c}$ for all $1 \leqslant k<l \leqslant w, I_{\mathbf{j}}=\left\{H_{j_{1}}^{c}, \cdots, H_{j_{w}}^{c}\right\} \subseteq \bar{I}, \mathcal{I}_{\mathbf{j}}=\left\{S_{j_{1}}^{c}, S_{j_{2}}^{c}, \cdots, S_{j_{w}}^{c}\right\}$, $\mathbf{I}_{\mathbf{j}}=\left\{G_{b_{1}}\left|S_{j_{1}}^{c}, G_{b_{2}}\right| S_{j_{2}}^{c}, \cdots, G_{b_{w}} \mid S_{j_{w}}^{c}\right\}, G_{b_{k}} \mid S_{j_{k}}^{c}$ is closed for all $1 \leqslant k \leqslant w$. Then, $H_{i}^{c} \nless H_{j}^{c}$ for each $S_{j}^{c} \in G_{\mathcal{I}_{j}}^{*}$ and $H_{i}^{c} \in I$.

(ii) For all $H \in \bar{\tau}_{I}^{\mathcal{i}}$, let

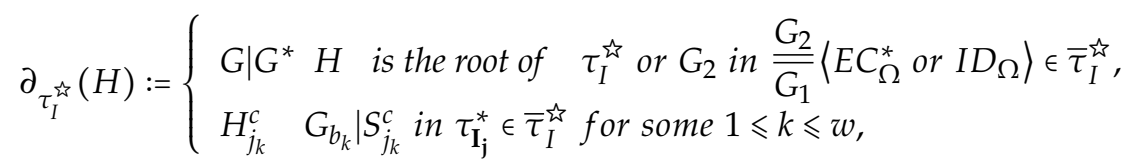

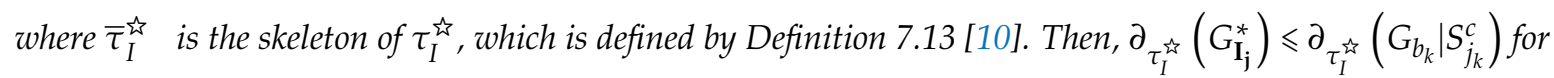
some $1 \leqslant k \leqslant w$ in $\tau_{\mathbf{I}^{\prime}}^{*}$

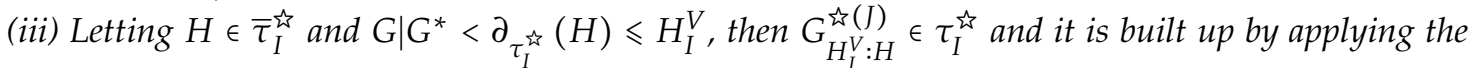
separation algorithm along $H_{I}^{V}$ to $H$, and is an upper hypersequent of either $\left\langle E C_{\Omega}^{*}\right\rangle$ if it is applicable, or $\left\langle I D_{\Omega}\right\rangle$, otherwise.

(iv) $S_{j}^{c} \in G_{I}^{i s}$ implies $H_{j}^{c} \| H_{i}^{c}$ for all $H_{i}^{c} \in I$ and, $S_{j}^{c} \in G_{\mathcal{I}_{\mathbf{j}}}^{*}$ for some $\tau_{\mathbf{I}_{\mathbf{j}}}^{*} \in \tau_{I}^{i \sim}$.

Proof. $\tau_{I}^{\tau_{3}}$ is constructed by induction on $o(I)$. For the base case, let $o(I)=1$; then, $\tau_{I}^{\tau^{2}}$ is built up by Construction 7.3 and 7.7 in [10]. For the induction case, suppose that $o(I) \geqslant 2, \tau_{I_{l}}^{\hbar_{L}}$ and $\tau_{I_{r}}^{\hbar_{r}}$ are constructed such that Claims from (i) to (iv) hold.

Let $\frac{G^{\prime}\left|S^{\prime} \quad G^{\prime \prime}\right| S^{\prime \prime}}{G^{\prime}\left|G^{\prime \prime}\right| H^{\prime}}(I I) \in \tau^{*}$, where $G^{\prime}\left|G^{\prime \prime}\right| H^{\prime}=H_{I}^{V}$. Then, $I_{l}$ and $I_{r}$ occur in the left subtree $\tau^{*}\left(G^{\prime} \mid S^{\prime}\right)$ and right subtree $\tau^{*}\left(G^{\prime \prime} \mid S^{\prime \prime}\right)$ of $\tau^{*}\left(H_{I}^{V}\right)$, respectively. Here, almost all manipulations of the new main algorithm are the same as those of the old main algorithm. There are some caveats that need to be considered.

Firstly, all leaves $\overline{\overline{G \mid G^{*}}}\left\langle\tau^{*}\right\rangle \in \bar{\tau}_{I_{l}}^{\tau_{2}}$ are replaced with $\tau_{I_{r}}^{\text {出 }}$ in Step 3 at Stage 1 in the old main algorithm and $\overline{\overline{G \mid G^{*}}}\left\langle\tau^{*}\right\rangle \in \bar{\tau}_{I_{r}}^{\hbar_{3}}$ are replaced with $\tau_{I_{l}}^{\text {出}}$ in Step 3 at Stage 2. Secondly, we abandon the definitions of branch to $I$ and Notation 8.1 in [10] and then the symbol I of the set of branches, which occur in $\tau_{\mathbf{I}}^{\text {is }}$ in [10], is replaced with $I$ in the new algorithm. We call the new algorithm the separation algorithm along $I$. We also replace $\Omega$ in $\tau_{\mathbf{I}}^{\Omega}$ with is. Thirdly, under the new requirement that $I$ is complete, we prove the following property.

Property (A) $G_{I_{l}}^{\text {is }}$ contains at most one copy of $G_{H_{I}^{V}: G^{\prime \prime}}^{i s}\left(\widehat{S^{\prime \prime}}\right.$.

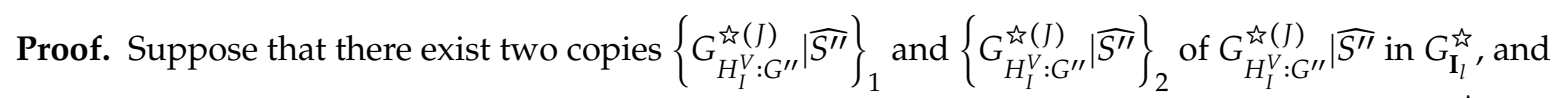

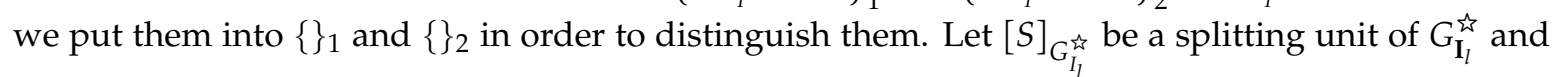
$S$ its splitting sequent. Then, $\left|v_{l}(S)\right|+\left|v_{r}(S)\right| \geqslant 2$. Thus, $S$ is a $(p E C)$-sequent and has the form $S_{i}^{c}$

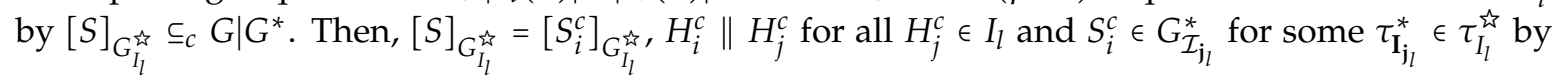
Claim (iv). Since $I_{l}$ is complete and $G^{\prime} \mid S^{\prime} \leqslant H_{I_{l}}^{V}$, then $H_{i}^{c} \| G^{\prime} \mid S^{\prime}$. 
Let $\tau_{\mathbf{I}_{\mathbf{j}_{l}}}^{*}$ be in the form $\frac{G_{b_{l 1}}\left|S_{j_{l_{1}}}^{c} G_{b_{l 2}}\right| S_{j_{l 2}}^{c} \cdots G_{b_{l u}} \mid S_{j_{l u}}^{c}}{G_{\mathbf{I}_{\mathbf{j}_{l}}}^{*}=\left\{G_{b_{l k}}\right\}_{k=1}^{u} \mid G_{\mathcal{I}_{\mathbf{j}_{l}}}^{*}}\left|\tau_{\mathbf{I}_{\mathbf{j}_{l}}}^{*}\right\rangle, \frac{G_{1}\left|S_{1} G_{2}\right| S_{2}}{H_{1} \equiv G_{1}\left|G_{2}\right| H^{\prime \prime}}(I I) \in \tau^{*}$, where $G_{1} \mid S_{1} \leqslant$ $G^{\prime}\left|S^{\prime}, G_{2}\right| S_{2} \leqslant H_{i}^{c}, G_{1}\left|G_{2}\right| H^{\prime \prime}$ is the intersection node of $H_{i}^{c}$ and $G^{\prime} \mid S^{\prime}$, as shown in Figure 3 . Then, $\frac{\left\{G_{b_{l k}}\right\}_{k=1}^{u}\left|\left\langle G_{1} \mid S_{1}\right\rangle_{\mathcal{I}_{\mathfrak{j}_{l}}} G_{2}\right| S_{2}}{H_{2} \equiv\left\{G_{b_{l k}}\right\}_{k=1}^{u}\left|\left\langle G_{1}\right\rangle_{\mathcal{I}_{\mathbf{j}_{l}}}\right| G_{2} \mid H^{\prime \prime}}(I I) \in \tau_{\mathbf{I}_{\mathbf{j}_{l}}}^{*}$ by $G_{1}\left|S_{1} \leqslant G^{\prime}\right| S^{\prime} \leqslant H_{I_{l}}^{V}$ and $S_{i}^{c} \in G_{\mathcal{I}_{\mathfrak{j}_{l}}}^{*}$. Since $S_{2}$ is separable in $G_{I_{l}}^{\text {is }}$ by $G^{\prime} \mid S^{\prime} \leqslant H_{I_{l}}^{V}$, then $S_{i}^{c} \in G_{2} \mid S_{2}$ and $S_{i}^{c}$ is not $S_{2}$.

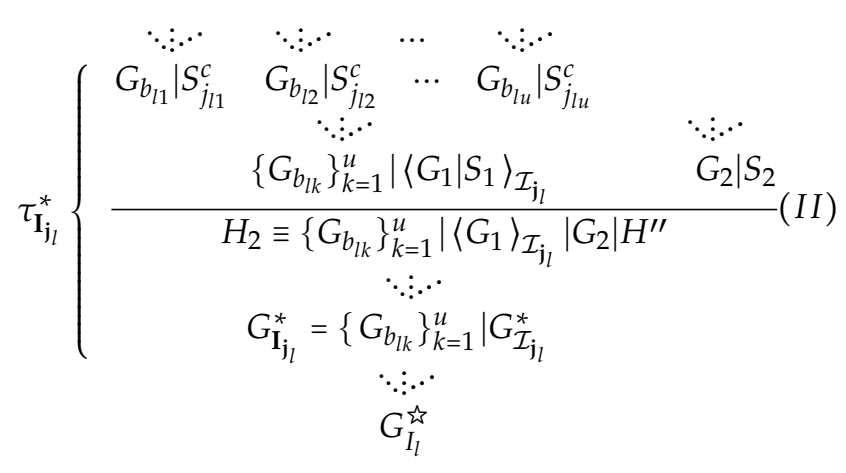

Figure 3. A fragment of $\tau_{I_{l}}^{\text {色. }}$.

Before proceeding to prove Property (A), we present the following property of $\left[S_{i}^{c}\right]_{G_{I_{l}}^{\text {th }}}$.

Property (B) The set of splitting sequents of $\left[S_{i}^{c}\right]_{G_{I_{l}}^{\vec{b}}}$ is equal to that of $\left[S_{i}^{c}\right]_{G_{2} \mid S_{2}}$.

Proof. Let $\frac{G_{1}^{\prime}\left|S_{1}^{\prime} \quad G_{2}^{\prime}\right| S_{2}^{\prime}}{H_{1}^{\prime} \equiv G_{1}^{\prime}\left|G_{2}^{\prime}\right| H^{\prime \prime \prime}}(I I) \in \tau^{*}, G_{1}^{\prime} \mid S_{1}^{\prime} \leqslant H_{1}$ and $S_{1}^{\prime} \in\left\langle G_{1}^{\prime} \mid S_{1}^{\prime}\right\rangle_{\mathcal{I}_{\mathbf{j}_{l}}}$. Then, $S_{1}^{\prime}$ and $S_{2}^{\prime}$ are separable

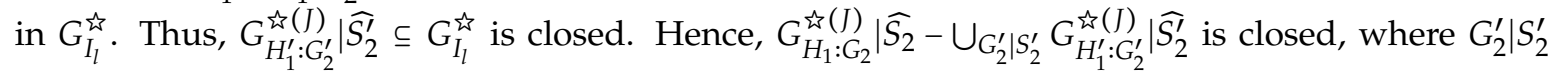
in $\bigcup_{G_{2}^{\prime} \mid S_{2}^{\prime}}$ runs over all $I I \in \tau^{*}$ above such that $G_{H_{1}^{\prime}: G_{2}^{\prime}}^{i j(J)} \widehat{S_{2}^{\prime}} \subseteq G_{H_{1}: G_{2}}^{i j(J)} \mid \widehat{S_{2}}$. Therefore, $v\left(G_{H_{1}: G_{2}}^{i j(J)} \mid \widehat{S_{2}}-\right.$ $\left.\cup_{G_{2}^{\prime} \mid S_{2}^{\prime}} G_{H_{1}^{\prime}: G_{2}^{\prime}}^{\mathcal{L}(J)} \mid \widehat{S_{2}^{\prime}}\right)=v\left(G_{2} \mid S_{2}\right),\left\{S_{j}^{c}: S_{j}^{c} \in G_{2}\left|S_{2}, H_{j}^{c} \geqslant G_{2}\right| S_{2}\right\}=\left\{S_{j}^{c}: S_{j}^{c} \in G_{H_{1}: G_{2}}^{i j}(J)\left|\widehat{S_{2}}-\cup_{G_{2}^{\prime} \mid S_{2}^{\prime}} G_{H_{1}^{\prime}: G_{2}^{\prime}}^{i j(J)}\right| \widehat{S_{2}^{\prime}}\right\}$

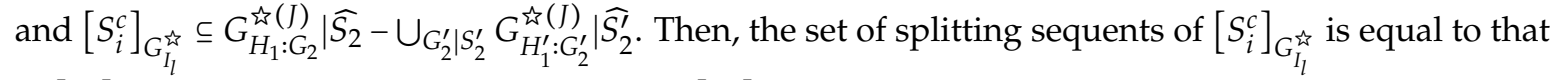
of $\left[S_{i}^{c}\right]_{G_{2} \mid S_{2}}$ since each splitting sequent $S^{\prime \prime \prime} \in\left[S_{i}^{c}\right]_{G_{I_{l}}^{\text {ch }}}$ is a $(p E C)$-sequent by $\left|v_{l}\left(S^{\prime \prime \prime}\right)\right|+\left|v_{r}\left(S^{\prime \prime \prime}\right)\right| \geqslant 2$ and $S^{\prime \prime \prime} \epsilon_{c} G \mid G^{*}$. This completes the proof of Property (B).

We therefore assume that, without loss of generality, $S_{i}^{c}$ is in the form $\Gamma, p_{k}, \Delta \Rightarrow p_{k}$ by Property (B), Lemma 5 and the observation that each derivation-splicing operation is local. There are two cases to be considered in the following.

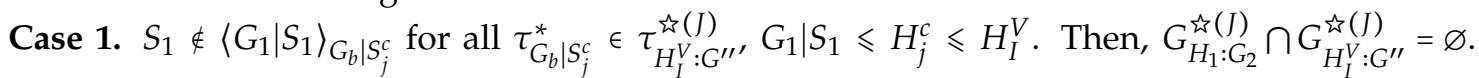
We assume that, without loss of generality, $\left\langle G_{2} \mid S_{2}\right\rangle_{k}^{-}=G_{2}^{\prime}\left|\Gamma \Rightarrow t,\left\langle G_{2} \mid S_{2}\right\rangle_{k}^{+}=G_{2}^{\prime \prime}\right| S_{2} \mid \Delta \Rightarrow t$. Then, $\left\langle G_{I_{l}}^{i \hat{j}}\right\rangle_{k}^{-}=G_{H_{2}: G_{2}^{\prime}}^{i j(J)} \mid \Gamma \Rightarrow t$ since $S=\Gamma, p_{k}, \Delta \Rightarrow p_{k}$ isn't a focus sequent at all nodes from $G_{2} \mid S_{2}$ to $G_{I_{l}}^{\text {is }}$ in $\tau_{I_{l}}^{\hbar_{j}^{j}}$ and, $H_{j}^{c} \leqslant H_{1}$ or $H_{j}^{c} \| G_{1} \mid S_{1}$ for all $S_{j}^{c} \in G_{2}^{\prime}$ by Lemma 6.7 in [10]. Thus,

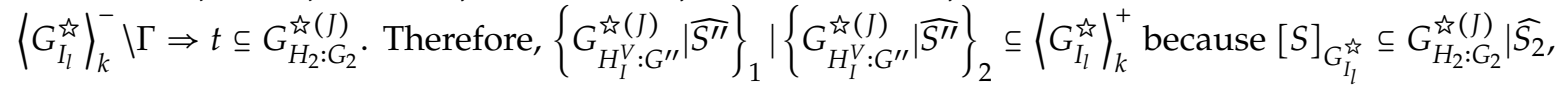

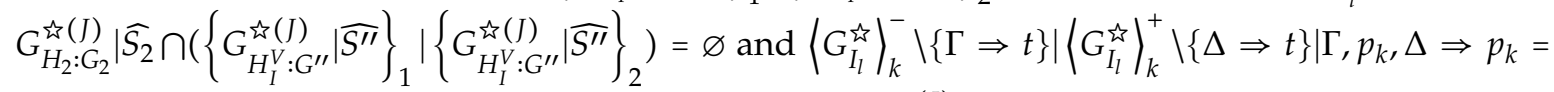

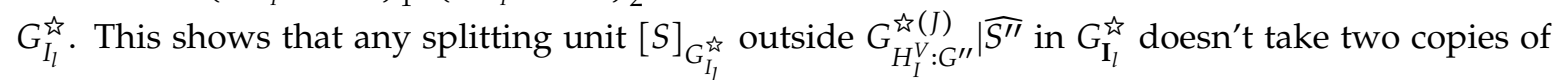

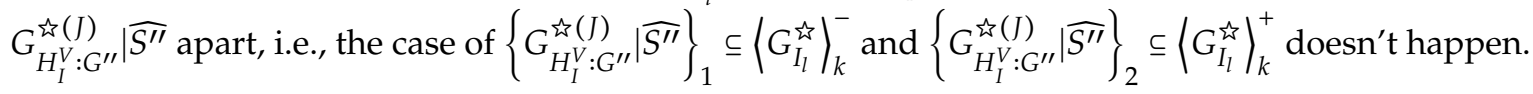


Case 2. $S_{1} \in\left\langle G_{1} \mid S_{1}\right\rangle_{G_{b} \mid S_{j}^{c}}$ for some $\tau_{G_{b} \mid S_{j}^{c}}^{*} \in \tau_{H_{I}^{V}: G^{\prime \prime}}^{\xi(J)} G_{1} \mid S_{1} \leqslant H_{j}^{c} \leqslant H_{I}^{V}$. Then, $G_{b}\left|\left\langle G_{1}\right\rangle_{S_{j}^{c}}\right| G_{2} \mid H^{\prime \prime} \epsilon$

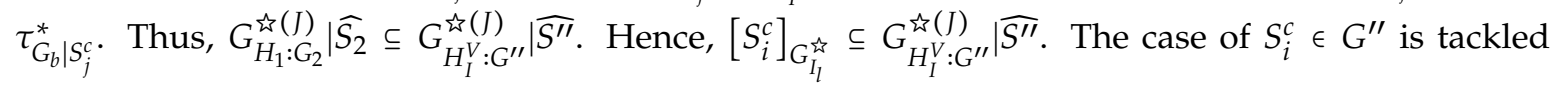
with the same procedure as the following. Let $\left[S_{i}^{c}\right]_{G_{I_{l}}^{\text {मे }}} \subseteq\left\{G_{H_{I}^{V}: G^{\prime \prime}}^{\text {th }(J)} \widehat{S^{\prime \prime}}\right\}_{1}$. Then, there exists a copy of $[S]_{G_{I_{l}}^{\text {r }}}$ in $\left\{G_{H_{I}^{V}: G^{\prime \prime}}^{i(J)} \widehat{S^{\prime \prime}}\right\}_{2}$ and let $\Gamma, p_{k^{\prime}}, \Delta \Rightarrow p_{k^{\prime}}$ be its splitting sequent. We put two splitting units

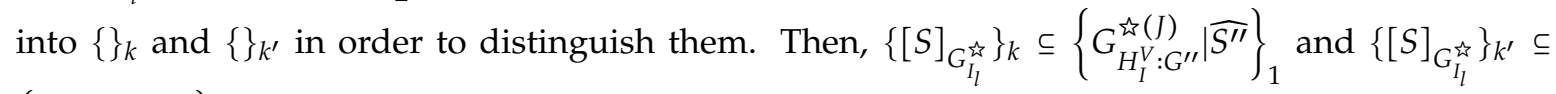
$\left\{G_{H_{I}^{V}: G^{\prime \prime}}^{i \sim}(J) \mid \widehat{S^{\prime \prime}}\right\}_{2}$. We assume that, without loss of generality, $\left\langle G_{2} \mid S_{2}\right\rangle_{k}^{-}=G_{2}^{\prime}\left|\Gamma \Rightarrow t,\left\langle G_{2} \mid S_{2}\right\rangle_{k}^{+}=G_{2}^{\prime \prime}\right| S_{2} \mid \Delta \Rightarrow t$.

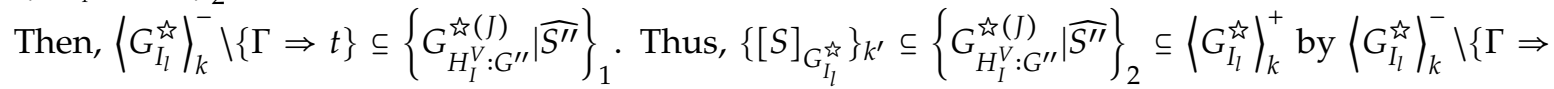

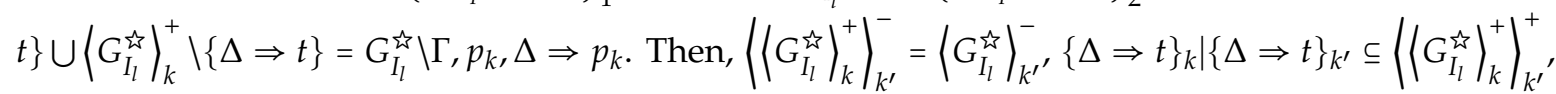
where we put two copies of $\Delta \Rightarrow t$ into \{\}$_{k}$ and \{\}$_{k^{\prime}}$ in order to distinguish them. Then, $\Gamma \Rightarrow t \in\left\langle G_{I_{l}}^{\sharp i}\right\rangle_{k^{\prime}}^{-}$

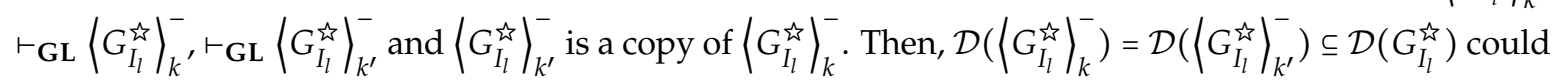
be cut off of one of them because they are the two same sets of hypersequents in $\mathcal{D}\left(G_{I_{l}}^{\text {th }}\right)$. Meanwhile, two copies of $\Delta \Rightarrow t$ in $\left\langle\left\langle G_{I_{l}}^{i j}\right\rangle_{k}^{+}\right\rangle_{k^{\prime}}^{+}$can't be taken apart by any splitting unit outside $G_{H_{I}^{V}: G^{\prime \prime}}^{\text {in }(J)} \widehat{S^{\prime \prime}}$ in $G_{I_{l}}^{\text {in }}$ for the reason as shown in Case 1 and thus could be contracted into one by $(E C)$ in $\mathcal{D}\left(G_{I_{l}}^{\text {i⿱ }}\right)$. Therefore,

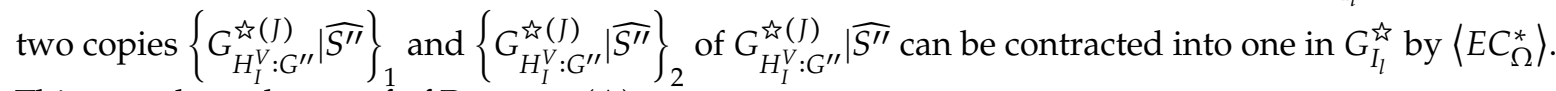
This completes the proof of Property (A).

With Property (A), all manipulations in the old main algorithm in [10] work well. This completes the construction of $\tau_{I}^{i /}$ and the proof of Theorem 4 .

Theorem 5. The standard completeness holds for $\mathbf{H p s U L}^{*}$.

Proof. Let $\stackrel{i}{\longleftrightarrow}$ denote the $i$-th logical link of iff in the following. $\vDash \mathcal{K} A$ means that $v(A) \geqslant t$ for every algebra $\mathcal{A}$ in $\mathcal{K}$ and valuation $v$ on $\mathcal{A}$. Let $\mathbf{p s U L}{ }^{*}, \operatorname{LIN}\left(\mathbf{p s U L}^{*}\right), \mathbf{p s U L}^{* D}$ and $[0,1]_{\mathbf{p s U L}} \mathrm{L}^{*}$ denote the classes of all $\mathbf{p s U L}{ }^{*}$-algebras, $\mathbf{p s U L} \mathbf{L}^{*}$-chain, dense $\mathbf{p s} \mathbf{U L}^{*}$-chain and standard $\mathbf{p s U L ^ { * }}$-algebras (i.e., their lattice reducts are $[0,1])$, respectively. We have an inference sequence, as shown in Figure 4.

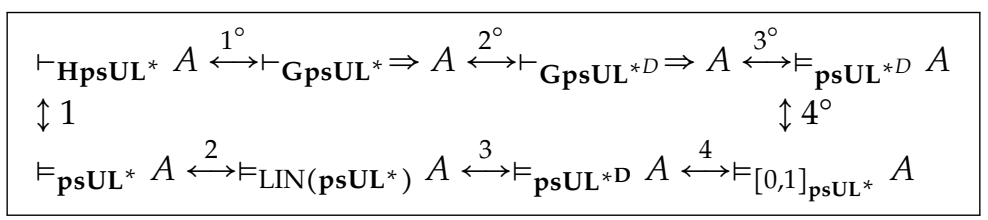

Figure 4. Two ways to prove standard completeness.

Links from 1 to 4 show Jenei and Montagna's algebraic method to prove standard completeness and, currently, it seems hopeless to build up link 3 (see [11-14]). Links from $1^{\circ}$ to $4^{\circ}$ show Metcalfe and Montagna's proof-theoretical method. Density elimination is at Link $2^{\circ}$ in Figure 4 and other links are proved by standard procedures with minor revisions and omitted (see [1,4,15-17]).

\section{Future Works}

Generally, for any existing fuzzy logic system, we can consider its corresponding non-commutative system, just as HpsUL is obtained by removing the commutativity of the strong conjunctive connective $\odot$ in UL. Therefore, we can consider the corresponding non-commutative 
systems of many systems. A natural question is whether the method of the density elimination proposed in this paper can be generalized to these systems. It has often been the case in the past that metamathematical methods have corresponding algebraic analogues. The method proposed in this paper is essentially proof-theoretic. A natural problem is whether there is an algebraic proof corresponding to our proof-theoretic one.

Funding: This research was funded by the National Foundation of Natural Sciences of China (Grant No: 61379018, 61662044, 11571013, and 11671358).

Conflicts of Interest: The authors declare no conflict of interest.

\section{References}

1. Metcalfe, G.; Olivetti, N.; Gabbay, D. Proof Theory for Fuzzy Logics; Springer Series in Applied Logic; Springer: Berlin, Germany, 2009; Volume 36, ISBN 9781402094095

2. Wang, S.M.; Zhao, B. HpsUL is not the logic of pseudo-uninorms and their residua. Log. J. IGPL 2009, 17, 413-419. [CrossRef]

3. Wang, S.M. Logics for residuated pseudo-uninorms and their residua. Fuzzy Sets Syst. 2013, 218, $24-31$. [CrossRef]

4. Metcalfe, G.; Montagna, F. Substructural fuzzy logics. J. Symb. Log. 2007, 7, 834-864. [CrossRef]

5. Moallem, P.; Mousavi, B.S.; Naghibzadeh, S.S. Fuzzy inference system optimized by genetic algorithm for robust face and pose detection. Int. J. Artif. Intell. 2015, 13, 73-88.

6. Jankowski, J.; Kazienko, P.; Watróbski, J.; Lewandowska, A.; Ziemba, P.; Zioło, M. Fuzzy multi-objective modeling of effectiveness and user experience in online advertising. Expert Syst. Appl. 2016, 65, 315-331. [CrossRef]

7. Precup, R.E.; Tomescu, M.L.; Preitl, Ş. Fuzzy logic control system stability analysis based on Lyapunov's direct method. Int. J. Comput. Commun. Control 2009, 4, 415-426. [CrossRef]

8. S̆krjanc, I.; Blažičc, S.; Matko, D. Direct fuzzy model-reference adaptive control. Int. J. Intell. Syst. 2002, 17, 943-963. [CrossRef]

9. Metcalfe, G.; Tsinakis, C. Density revisited. Soft Comput. 2017, 21, 175-189. [CrossRef]

10. Wang, S.M. Density Elimination for Semilinear Substructural Logics. arXiv 2015, arXiv:1509.03472. [CrossRef]

11. Jenei, S.; Montagna, F. A proof of standard completeness for Esteva and Godo's logic MTL. Stud. Log. 2002, 70, 183-192. [CrossRef]

12. Wang, S.M. Uninorm logic with the n-potency axiom. Fuzzy Sets Syst. 2012, 205, 116-126. [CrossRef]

13. Wang, S.M. Involutive uninorm logic with the $n$-potency axiom. Fuzzy Sets Syst. 2013, 218. [CrossRef]

14. Wang, S.M. The Finite Model Property for Semilinear Substructural Logics. Math. Log. Q. 2013, 59, 268-273. [CrossRef]

15. Ciabattoni, A.; Galatos, N.; Terui, K. Algebraic proof theory for substructural logics: Cut-elimination and completions. Ann. Pure Appl. Log. 2012, 163, 266-290. [CrossRef]

16. Nikolaos, G.; Jipsen, P.; Kowalski, T.; Ono, H. Residuated Lattices: An Algebraic Glimpse at Substructural Logics; Elsevier: Amsterdam, The Netherlands, 2007; ISBN 978-0-444-52141-5.

17. Baldi, P.; Terui, K. Densification of FL chains via residuated frames. Algebr. Univers. 2016, 75, 169-195. [CrossRef]

(C) 2019 by the author. Licensee MDPI, Basel, Switzerland. This article is an open access article distributed under the terms and conditions of the Creative Commons Attribution (CC BY) license (http://creativecommons.org/licenses/by/4.0/). 